\title{
Corner-Based Estimation of Tire Forces and Vehicle Velocities Robust to Road Conditions
}

\author{
Ehsan Hashemi , Mohammad Pirani, Amir Khajepour, Alireza Kasaiezadeh, Shih-Ken Chen, Bakhtiar Litkouhi
}

\begin{abstract}
Recent developments in vehicle stability control and active safety systems have led to an interest in reliable vehicle state estimation on various road conditions. This paper presents a novel method for tire force and velocity estimation at each corner to monitor tire capacities individually. This is entailed for more demanding advanced vehicle stability systems and especially in full autonomous driving in harsh maneuvers. By integrating the lumped LuGre tire model and the vehicle kinematics, it is shown that the proposed corner-based estimator does not require knowledge of the road friction and is robust to model uncertainties. The stability of the time-varying longitudinal and lateral velocity estimators is explored. The proposed method is experimentally validated in several maneuvers on different road surface frictions. The experimental results confirm the accuracy and robustness of the state estimators.
\end{abstract}

Keywords-Vehicle state estimation, velocity estimation, tire force estimation, robust observer design

\section{INTRODUCTION}

Advanced vehicle stability control and active safety systems require dependable vehicle states, which may not be accessible by measurements and should be estimated. Two major practical issues that have dominated the vehicle state estimation field are velocity and tire force estimation robust to the road friction changes. Tire forces can be measured at each corner with sensors mounted on the wheel hub, but their significant cost, required space, and calibration and maintenance make them completely unfeasible for mass production vehicles. Provided that the tire force calculation needs road friction, even accurate slip ratio/angle information from the GPS will not engender forces at each corner. Hence, estimation of the longitudinal and lateral tire forces would be a remedy. Estimation of longitudinal and lateral forces independent from the road condition may be classified on the basis of wheel dynamics into the nonlinear and sliding mode observers [1]-[3], Kalmanbased estimation [4]-[6], and unknown input observers [7][9]. A force estimation method based on the steering torque measurement is introduced in [10], [11], which requires additional measurements. A high gain observer with inputoutput linearization is proposed by Gao et al. [12] to estimate the lateral states. An extended Kalman filter (EKF) is employed in [13] to estimate tire forces and road friction condition.

Longitudinal and lateral velocities make major contributions to traction and stability control systems, respectively. They can be measured with GPS, but the poor accuracy of the

Ehsan Hashemi, Mohammad Pirani, and Amir Khajepour are with the Department of Mechanical and Mechatronics Engineering, University of Waterloo, ON, Canada. email: ehashemi, mpirani, a.khajepour@uwaterloo.ca

Alireza Kasaiezadeh, Shih-Ken Chen, and Bakhtiar Litkouhi are with the R\&D Department, General Motors Co., Warren, MI, USA mostly practiced conventional GPSs and the loss of reception in some areas are primary drawbacks. Literature has adopted three major approaches for longitudinal/lateral velocity estimation. One is the modified kinematic-based approach, which uses acceleration and the yaw rate measurements from an inertial measurement unit (IMU) and estimates the vehicle velocities employing Kalman-based [14], [15], or nonlinear [16] observers. This method does not employ a tire model, but instead the sensor's bias and noise should be identified precisely to have a reliable estimation. In addition, lowexcitation cases that lead to erroneous estimation should be handled with this method. To increase the accuracy of the estimated heading and position, Farrell et al. [17] used the carrier-phase differential GPS, which requires a base tower and increases the cost significantly. To remove noises and address the low excitation scenarios, some kinematic-based methodologies [18], [19] employ accurate GPS, which may be lost and impose additional costs on commercial vehicles. Yoon and Peng [20] utilize two low-cost GPS receivers for the lateral velocity estimation and compensate the low update rate issue of conventional GPS receivers by combining the IMU and GPS data using an EKF.

The other velocity estimation method integrates measured longitudinal/lateral accelerations and uses an observer on tire forces to correct the estimation. This approach requires a good perception of the road friction and a precise tire model. To deal with the varying tire parameters and model uncertainties, model scheduling is introduced in [21] and [22] using tire slips. A nonlinear observer is also provided in [23] with simultaneous bank angle estimation to address the unknown tire parameters. An EKF is employed for both longitudinal and lateral vehicle state estimations in [24], [25]. EKF has been used in [26] along with the Burckhardt model [27] to estimate the vehicle states and tire model parameters; an EKF with smooth variable structure is also utilized in [28] to estimate lateral velocities and sideslip angles. Computational complexities of the EKF justify using a reliable approach such as the unscented Kalman filter (UKF) [29] without any need for linearization in system dynamics. Antonov et al. employ an unscented Kalman filter for vehicle state estimation in [30] and provided a longitudinal/lateral velocity estimator at each corner. They utilized wheel torques, wheel speeds, and a simplified empirical Magic formula [31] as the tire model, which requires known tire parameters and road friction. Similarly, employing UKF, Wielitzka et al. [32] and Sun et al. [33] propose different methods for estimation of the lateral and longitudinal velocities using knowledge of the road condition, Magic formula, and LuGre [34] tire models respectively.

On the other hand, to address unknown road friction, 
some literature attempted to identify the road condition and estimated vehicle states simultaneously. Grip et al. suggest a nonlinear sideslip angle observer in [35] and [36] that incorporates time-varying gains and estimates the vehicle states as well as the surface friction using a tire model. Their method should cope with the noises and uncertainties imposed by road identification errors due to the lack of excitation. You et al. [37] introduce an adaptive least square approach to jointly estimate the lateral velocities and tires' cornering stiffness (road friction terms). The road bank angle is also identified in their approach. However, lateral acceleration measurement noises have not been addressed. A sliding-mode observer is provided by Magallan et al. in [38] based on the LuGre tire model [34] to estimate the longitudinal velocity and the surface friction. Zhang et al. propose a sliding-mode observer in [39] to estimate velocities using wheel speed sensors, braking torque and longitudinal/lateral acceleration measurements. Their approach utilizes a sliding-mode observer for the velocity estimation and an EKF for estimation of the Burckhardt tire model's friction parameter. However, this method needs accurate tire parameters in presence of tire wear, inflation pressure, and road uncertainties. A switched nonlinear observer based on a simplified Pacejka tire model is introduced by Sun et al. [40] to provide estimates of longitudinal and lateral vehicle velocities and the tire-road friction coefficient during anti-lock braking. Their approach benefits from switching in specific cases because of unreliability of the measurements, but it relies on a predefined zero slip ratio for the longitudinal velocity measurement.

Other studies focus on the velocity estimation robust to the road condition, but implements additional measurements which are not common for conventional cars or require identification of tire parameters. Hsu et al. propose a method in [10] and [41] to estimate the road friction condition and sideslip angle using the steering torque sensor, which may not be applicable for all production vehicles. Nam et al. [42] present a sideslip angle estimation method with a recursive least squares algorithm to improve stability of in-wheel-motor-driven electric vehicles, but their approach uses force measurements from the multisensing hub units, which are not available for all electric and conventional cars. Gadola et al. investigate a Kalman-based lateral vehicle estimation on a single-track car model in [43] with the Magic formula tire model. The derivatives of the lateral forces in their approach, however, may amplify noise effects in the lateral/longitudinal state estimates.

Therefore, developing an observer-based vehicle state estimator using conventional sensor measurement (wheel speed, steering angle, and IMU) without using road friction information is desirable and provided in this paper.

\section{Proposed Corner-BAsed Estimation Structure}

This paper provides different observers for the vehicle state estimation on various road friction with the following distinct contributions:

- Longitudinal, lateral, and vertical tire-free force estimators are developed using computationally efficient nonlinear observers and common measurements without any limiting assumption on the lateral force distribution proportional to the vertical loads on a track.

- A novel parameter-varying observer for the velocity estimation, robust to road friction changes, is introduced which treats acceleration measurement noises and the road condition as uncertainties.

- The newly introduced velocity estimator can be utilized in many road identification approaches [3], [13], [44], [45]. However, those references usually employ the slip ratio/angle measurement from accurate GPS data, that is not available for production vehicles.

- The corner-based structure of the longitudinal/lateral force and velocity estimators advantageously leads to better performance of the stability and traction control systems which need slip ratio/angle at each corner

- The combined kinematic and model-based algorithm for estimation of the sideslip angle and slip ratio has a modular structure which can employ any force estimation module.

The corner-based state estimation structure is illustrated in Fig. 1, in which the estimations from Longitudinal Force Est and Lateral Force Est modules are fed to the observerbased velocity estimators. Longitudinal, lateral, and vertical force estimators in this article are developed using nonlinear and Kalman-based observers. The longitudinal force estimators uses total torques and wheel speeds at each corner. The UKFbased lateral force estimator employs vehicle lateral dynamics, acceleration measurements, steering, and the yaw rate. Vertical forces at each corner from Normal Force Est are obtained by load transfer, vehicle angles and accelerations; vertical forces are utilized for normalization of the longitudinal and lateral forces in the velocity estimators. In the newly proposed Longitudinal Velocity Est and Lateral Velocity Est modules, kinematic-based approach is combined with the internal tire states considering road friction and measurement noises as uncertainties. The longitudinal and lateral velocity estimators use accelerations, yaw rate, steering angle, roll dynamics, estimated tire forces, and provide slip angle/ratio at each tire. Measured accelerations by IMU attached to the sprung mass are corrected with the vehicle's body pitch and roll angles from Pitch/Roll Angle Est to include only the kinematics of the motion. These corrected values are then used for the normal force and velocity estimators.

Finally, the proposed approach is experimentally validated on various road conditions using a full-size test vehicle.

In the following, a corner-based methodology for the longitudinal, lateral, and normal force estimation is first discussed in section III. The suggested observer-based longitudinal and lateral velocity estimator is then provided in section IV. The stability and performance of the linear parameter-varying error dynamic system is also investigated in this section. Section V presents experimental results used to verify the approach on different road conditions and in various maneuvers with high and low longitudinal/lateral excitations. Finally, conclusions are provided. 


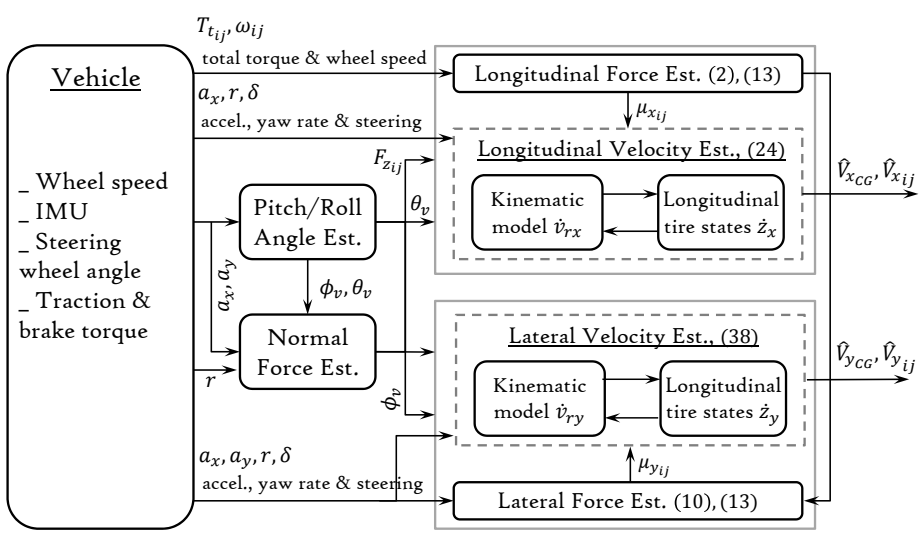

Fig. 1: Corner-based state estimation structure

\section{FORCE ESTIMATION}

An observer for the longitudinal force and an unscented Kalman filter for the lateral force estimation are designed in this section. Vertical forces are estimated using vehicle body dynamics, the load transfer, and the acceleration measurements.

\section{A. Longitudinal force estimation}

Longitudinal force estimation significantly contributes to vehicle stability control. When the total torque at each wheel is available, wheel dynamics can be utilized for the force estimation. Neglecting the bearing's viscous damping, one can write the wheel dynamic equation at each corner as (1) in the coordinates attached to the tires: (Fig. 2):

$$
F_{x_{i j}}=\frac{1}{R_{e}}\left(T_{t_{i j}}-I_{w} \dot{\omega}_{i j}\right)+\Omega_{f},
$$

where $R_{e}$ is the wheel effective radius, $T_{t_{i j}}$ represents the total effective torque on each wheel, $F_{x_{i j}}$ is the longitudinal tire force at each corner in the coordinate frame attached to the tires [46], $\dot{\omega}_{i j}$ is the wheel acceleration, $I_{w}$ is the wheel's moment of inertia and $\Omega_{f}$ represents uncertainties in the model including the wheel acceleration, torque, etc. A corner-based estimation approach proposed in this section for the longitudinal force estimation uses a PID state observer [47]-[50] that has also been used in other applications. The suggested estimator uses wheel torques $T_{t_{i j}}$ and speed $\omega_{i j}$ at each corner and provide an estimated wheel speed $\hat{\omega}_{i j}$ to estimate longitudinal tire force:

$$
\hat{F}_{x_{i j}}=\frac{T_{t_{i j}}-I_{w} \dot{\omega}_{i j}}{R_{e}}-\eta_{1} e_{\omega_{i j}}+\eta_{3} \int e_{F_{i j}} d t .
$$

where $i \in\{f, r\}$ (front and rear axles), $j \in\{L, R\}$ (left and right tires), $\eta_{1}, \eta_{3}$ are design parameters, and the estimated wheel speed error is $\left(e_{\omega_{i j}}=\omega_{i j}-\hat{\omega}_{i j}\right)$ where $\hat{\omega}_{i j}$ will be described in the following. Subtracting the longitudinal force (1) from the estimated force (2), the longitudinal force estimation error $e_{F_{i j}}=F_{x_{i j}}-\hat{F}_{x_{i j}}$ can be described as
$e_{F_{i j}}=-\eta_{3} \int e_{F_{i j}} d t+\eta_{1} e_{\omega_{i j}}+\Omega_{f}$. The time derivative of the error dynamic yields:

$$
\dot{e}_{F_{i j}}=-\eta_{3} e_{F_{i j}}+\eta_{1} \dot{e}_{\omega_{i j}}+\dot{\Omega}_{f}
$$

where the estimated wheel speed $\hat{\omega}$ at each corner $i j$ is described as:

$$
\dot{\hat{\omega}}_{i j}=\frac{1}{I_{w}}\left(T_{t_{i j}}-R_{e} \hat{F}_{x_{i j}}+\eta_{2} \int e_{\omega_{i j}} d t+R_{e} \eta_{3} \int e_{F_{i j}} d t\right),
$$

in which $\eta_{2}$ is another design parameter for this observer. The estimated wheel speed $\hat{\omega}_{i j}$ is an auxiliary term for longitudinal force estimation. Substituting $\hat{F}_{x_{i j}}$ from (2) in (4), one can rewrite:

$$
\dot{\hat{\omega}}_{i j}=\dot{\omega}_{i j}+\frac{1}{I_{w}}\left(R_{e} \eta_{1} e_{\omega_{i j}}+\eta_{2} \int e_{\omega_{i j}} d t\right) .
$$

The deviation of the estimated wheel speed from the measured one is denoted by $e_{\omega}$ and incorporated for the force estimation as in (2). In the implementation setup, the term $\int e_{F_{i j}} d t$ is attainable by the updated measurement $\frac{T_{t_{i j}}-I_{w} \dot{\omega}_{i j}}{R_{e}}$ at the current time step and the longitudinal force estimates $\hat{F}_{x_{i j}}$ from the previous time step, due to the fact that the uncertainty term $\Omega_{f}$ is mostly contributed to the wheel torque which is zero mean, its integral over time will eliminate the effect of $\Omega_{f}$.

Proposition 1: The estimation error for the longitudinal force estimator (2) is asymptotically bounded by $\sup _{t \geq 0} \frac{\left|\dot{\Omega}_{f}\right|}{\eta_{3}}$.

Proof: : Subtracting the estimated wheel speed (4) from the speed by the wheel dynamics (1), i.e., $\dot{\omega}_{i j}=$ $\frac{1}{I_{w}}\left[T_{t_{i j}}-R_{e}\left(F_{x_{i j}}-\Omega_{f}\right)\right]$, results in $I_{w} \dot{e}_{\omega_{i j}}=-R_{e} e_{F_{i j}}+$ $R_{e} \Omega_{f}-\eta_{2} \int e_{\omega_{i j}}-R_{e} \eta_{3} \int e_{F_{i j}} d t$. Taking time derivative and replacing the error dynamics (3) yield:

$$
I_{w} \ddot{e}_{\omega_{i j}}+R_{e} \eta_{1} \dot{e}_{\omega_{i j}}+\eta_{2} e_{\omega_{i j}}=0,
$$

which is in the following state-space form

$$
\left[\begin{array}{l}
\dot{e}_{\omega_{i j}} \\
\ddot{e}_{\omega_{i j}}
\end{array}\right]=\underbrace{\left[\begin{array}{cc}
0 & 1 \\
\frac{-\eta_{2}}{I_{w}} & \frac{-R_{e} \eta_{1}}{I_{w}}
\end{array}\right]}_{\mathcal{K}}\left[\begin{array}{l}
e_{\omega_{i j}} \\
\dot{e}_{\omega_{i j}}
\end{array}\right],
$$

where $\mathcal{K}$ is Hurwitz and (7) is exponentially stable provided $\eta_{1}, \eta_{2}>0$, therefore, $e_{\omega_{i j}} \rightarrow 0$ and $\dot{e}_{\omega_{i j}} \rightarrow 0$. Thus, the estimation error dynamics (3) asymptotically turns to

$$
\dot{e}_{F_{i j}}=-\eta_{3} e_{F_{i j}}+\dot{\Omega}_{f},
$$

that is an exponentially stable dynamic for $\forall \eta_{3}>0$. By solving (8) we get $e_{F_{i j}}(t)=e^{-\eta_{3} t} e_{F_{i j}}(0)+\frac{\dot{\Omega}_{f}}{\eta_{3}}$. As $e^{-\eta_{3} t} e_{F_{i j}}(0)$ exponentially converges to zero, $e_{F_{i j}}(t)$ will be asymptotically bounded by $\sup _{t \geq 0} \frac{\left|\dot{\Omega}_{f}\right|}{\eta_{3}}$.

Based on several road experiments on various road frictions, the designed observer has the gain $\eta_{3}=50.6$ and the order of $\dot{\Omega}_{f}$ is obtained as $\dot{\Omega}_{f}=\mathcal{O}\left(10^{2}\right)$. Other observer gains are provided in section V. Longitudinal force estimates (2) will be normalized by (13) to be implemented in the longitudinal velocity estimator (24). 


\section{B. Lateral force estimation}

Lateral tire forces are required for the vehicle stability control systems as well as the lateral velocity estimators. Lateral forces are estimated in this paper employing an unscented Kalman filter (UKF), using acceleration and yaw rate measurements, and vehicle's lateral dynamics. Figure 2 depicts longitudinal/lateral forces and slip angles at each corner in the $x y z$ coordinates attached to the vehicle body for the doubletrack vehicle model used in this study.
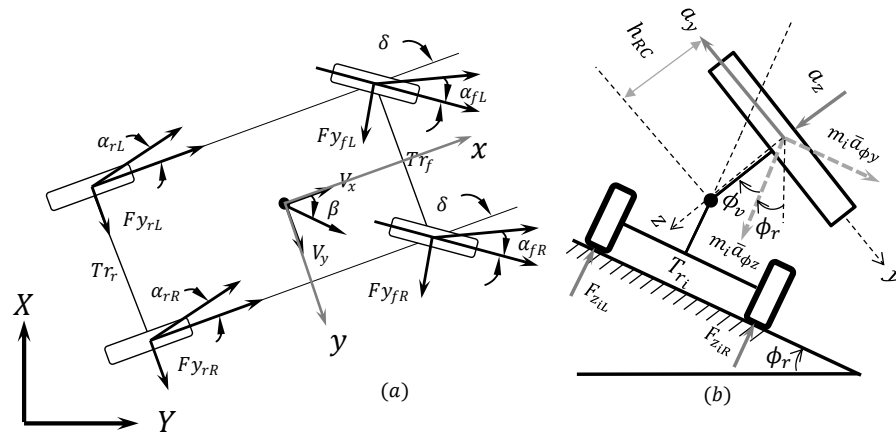

Fig. 2: (a) Double track vehicle model (b) Planar roll model

Introducing $F_{x_{i}}=\sum_{j} F_{x_{i j}}, F_{y_{i}}=\sum_{j} F_{y_{i j}}$ (the sum of longitudinal/lateral forces at each axle), vehicle's lateral dynamics with the measured acceleration and yaw rate in the body-fixed coordinates $x y z$ can be expressed as:

$$
\begin{aligned}
m a_{x} & =F_{x_{f}} \cos \delta-F_{y_{f}} \sin \delta+F_{x_{r}}, \\
m a_{y} & =F_{y_{f}} \cos \delta+F_{x_{f}} \sin \delta+F_{y_{r}}, \\
I_{z} \dot{r} & =\left(F_{y_{f}} \cos \delta+F_{x_{f}} \sin \delta\right) L_{f} \\
& +\left(\bar{F}_{x_{f}} \cos \delta-\bar{F}_{y_{f}} \sin \delta\right) \frac{T_{r_{f}}}{2}-F_{y_{r}} L_{r}+\bar{F}_{x_{r}} \frac{T_{r_{r}}}{2},
\end{aligned}
$$

in which $\delta$ is the steering angle (with parallel steering in front wheels). Distances from the front and rear axles' to CG are denoted by $L_{f}, L_{r}$ and $\bar{F}_{x_{i}}=F_{x_{i R}}-F_{x_{i L}}, \bar{F}_{y_{i}}=F_{y_{i R}}-$ $F_{y_{i L}}$. Longitudinal forces at each corner $F_{x_{i j}}$ are assumed to be available from (2) in the previous subsection. The set of equations (9) can be solved for the lateral forces $F_{y_{i j}}$ at each corner with the assumption of lateral force distribution based on the normal forces, but this may not address maneuvers in which road friction under each tire is different. To resolve this, an estimation method for lateral forces is proposed in this section that uses longitudinal forces, accelerations $a_{x}, a_{y}$, and the yaw rate $r$ measurements from a 3-axis IMU. The measured longitudinal and lateral accelerations include the kinematics of the vehicle's CG $V_{x}, V_{y}$, vehicle's body pitch/roll angles $\theta_{v}, \phi_{v}$, and road grade/bank angles $\theta_{r}, \phi_{r}$ as in $a_{x}=\dot{V}_{x}-$ $r V_{y}+g \sin \left(\theta_{v}+\theta_{r}\right)$ and $a_{y}=\dot{V}_{y}+r V_{x}+g \sin \left(\phi_{v}+\phi_{r}\right)$. The vehicle and road angles are estimated by an unknown input observer in [51].

The set of equations (9) can be rewritten in the following form to obtain the newly introduced lateral force estimator:

$$
\begin{aligned}
& \dot{\overline{\mathbf{x}}}=\bar{A} \overline{\mathbf{x}}+\bar{\Omega}, \\
& \overline{\mathbf{y}}=\underbrace{\left[\begin{array}{ccc}
-\frac{1}{m} \sin \delta & 0 & 0 \\
\frac{1}{m} \cos \delta & \frac{1}{m} & 0 \\
\frac{1}{I_{z}} \cos \delta L_{f} & -\frac{1}{I_{z}} L_{r} & \frac{1}{2 I_{z}} \sin \delta T_{r_{f}}
\end{array}\right]}_{\bar{C}(\delta)} \overline{\mathbf{x}} \\
& +\underbrace{\left[\begin{array}{c}
\frac{1}{m}\left(F_{x f} \cos \delta+F_{x r}\right) \\
\frac{1}{m} F_{x f} \sin \delta \\
\frac{1}{I_{z}}\left(F_{x f} \sin \delta L_{f}+\bar{F}_{x f} \cos \delta \frac{T_{r_{f}}}{2}+\bar{F}_{x r} \frac{T_{r_{r}}}{2}\right.
\end{array}\right]}_{\overline{\mathbf{u}}}+\overline{\boldsymbol{\Gamma}},
\end{aligned}
$$

where $\bar{A}=\mathbf{0}_{3 \times 3}$ and the states are $\overline{\mathbf{x}}=\left[\begin{array}{lll}F_{y_{f}} & F_{y_{r}} & \bar{F}_{y_{f}}\end{array}\right]^{T}$, output (measurement) is $\overline{\mathbf{y}}=\left[\begin{array}{lll}\bar{a}_{x} & \bar{a}_{y} & r\end{array}\right]^{T}$, and uncertainties in the process and measurements are denoted by $\overline{\boldsymbol{\Omega}}$ and $\overline{\boldsymbol{\Gamma}}$. Acceleration measurements $a_{x}, a_{y}$ are corrected with the road and body's roll/pitch angles as:

$$
\bar{a}_{x}=a_{x}-g \sin \left(\theta_{v}+\theta_{r}\right), \quad \bar{a}_{y}=a_{y}-g \sin \left(\phi_{v}+\phi_{r}\right) .
$$

Measured accelerations $a_{x}, a_{y}$ usually have bias and noises which can be addressed by a bias-removal method in high or low excitations or observer-based approaches [15]. The matrix $\bar{C}(\delta)$ in (10) is time-varying and physically bounded (because of the steering angle and its derivative). Thus, the observability matrix for the time-varying system (10) can be written as [52], [53]:

$$
\begin{array}{r}
\mathcal{O}_{n}=\left[\begin{array}{lll}
\xi_{1} & \xi_{2} \ldots & \xi_{n}
\end{array}\right]^{T} \\
\xi_{1}=\bar{C}, \quad \xi_{i+1}=\xi_{i} \bar{A}(t)+\dot{\xi}_{i},
\end{array}
$$

Observability of the system (10) is confirmed by holding the full rank condition $\operatorname{rank}\left(\mathcal{O}_{3}\right)=3$ for the operating regions of the steering angle and its time derivatives, except for the case where $\delta=0, k \pi$ for integer values of $k$. For the case where $\delta=0$ we have $\operatorname{rank}(\bar{C})=2$. However, in this case there is no lateral force applied to the tire. Moreover, situations $\delta=k \pi$ do not take place, due to the fact that the maximum value of the steering angle is much less than $\pi / 2$. Observability is a sufficient condition for implementation of an optimal variance filter (such as a Kalman estimator). Therefore, a Kalman-based observer can be employed on the discretized form of (10). The Gaussian and uncorrelated measurement/process noise are also required for such Kalman estimator. The unscented Kalman filter [29], [54] is used in this study to include non-Gaussian noises.

Remark 1: In general, discretization of the continuous-time system $\dot{x}=A_{c} x+B_{c} u$ with the output $y=C_{c} x+D_{c} u$ is done by the zero-order hold method [55], because of its precision and response characteristics. Input to the continuoustime system is the hold signal $u_{k}=u\left(t_{k}\right)$ for a period between $t_{k} \leq t<t_{k+1}$ with the sample time $T_{s}$. Then, the discretetime system has the output matrices $C_{d}=C_{c}, D_{d}=D_{c}$ and state/input matrices $A_{d}=e^{A_{c}(t) T_{s}}, B_{d}=\int_{0}^{T_{s}} e^{A_{c}(t) \tau} B_{c}(t) d \tau$ 
The unscented Kalman filter is utilized on the discretized from of (10) for the lateral force estimation at each corner. The UKF employs a transformation to include nonlinear characteristics of the system $\overline{\mathbf{x}}_{k+1}=\mathcal{F}\left(\overline{\mathbf{x}}_{k}, \overline{\mathbf{u}}_{k}, \overline{\boldsymbol{\Omega}}_{k}\right), \mathbf{y}_{k}=\mathcal{G}\left(\overline{\mathbf{x}}_{k}, \overline{\boldsymbol{\Gamma}}_{k}\right)$ with process and measurement uncertainties $\overline{\boldsymbol{\Omega}}_{k}, \overline{\boldsymbol{\Gamma}}_{k}$ and the covariance matrices $\bar{Q}_{k}=\mathbb{E}\left[\overline{\boldsymbol{\Omega}}_{k}, \overline{\boldsymbol{\Omega}}_{k}^{T}\right], \bar{R}_{k}=\mathbb{E}\left[\overline{\boldsymbol{\Gamma}}_{k}, \overline{\boldsymbol{\Gamma}}_{k}^{T}\right]$ in a recursive estimation procedure. The force estimator's process and measurements are $\overline{\mathbf{x}}_{k+1}=\bar{A}_{d} \overline{\mathbf{x}}_{k}+\overline{\mathbf{\Omega}}_{k}$ and $\mathbf{y}_{k}=\bar{C}_{d} \overline{\mathbf{x}}_{k}+\overline{\boldsymbol{\Gamma}}_{k}$ where $\bar{A}_{d}, \bar{C}_{d}$ are the state and output matrices of the discrete-time estimator (10).

The following procedure illustrates the lateral force estimation with UKF in which the proper capturing of nonlinearities contributed to the unscented transformation that defines Sigma vectors $\boldsymbol{\Sigma} \in \mathbb{R}^{N \times 2 N+1}$ ( $N$ is the length of the state vectors) around $\overline{\mathbf{x}}$. This propagation yields nonlinear stochastic characteristics of the random variables and results in getting the posterior mean and covariance up to second-order approximation [56], [57]. The square root factorization of the covariance matrix $\mathbf{P}_{k-1}$ is obtained by Cholesky decomposition at each time step $k$. Spread of the sigma points far from the mean values of random variables (states) are shown by the scalar $\bar{\tau}$. It is defined in [56] as $\bar{\tau}=\sqrt{N+\bar{\eta}}$, where $\bar{\eta}$ is the compound scaling parameter $\bar{\eta}=\bar{\epsilon}^{2} N-N$ and $\bar{\epsilon}=\sqrt{3 / N}$. Propagated sample points within the system $\mathcal{F}$ are represented by $\boldsymbol{\Sigma}_{k \mid k-1}$. The estimated function output is denoted by $\boldsymbol{\Lambda}_{k \mid k-1}$ and $W_{i}^{c}, W_{i}^{m}$ are weighting parameters defined by $W_{i}^{c}=W_{i}^{m}=\frac{1}{2}(N+\bar{\eta})$ for all sets $i \in\{1,2, \ldots, 2 N\}$ and $W_{0}^{c}=\frac{\bar{\eta}}{N+\bar{\eta}}+1-\bar{\epsilon}^{2}+\bar{\beta}, W_{0}^{m}=\frac{\bar{\eta}}{N+\bar{\eta}}$ for $i=0$. The parameter $\bar{\beta}=2$ is introduced to employ the prior information on the Gaussian distribution of $\overline{\mathbf{x}}$.

\section{LATERAL FORCE ESTIMATION, UKF}

Systems: (The estimator (10))

$\mathbf{x}_{k+1}=\mathcal{F}\left(\mathbf{x}_{k}, \mathbf{u}_{k}, \varrho_{q k}\right), \mathbf{y}_{k}=\mathcal{G}\left(\mathbf{x}_{k}, \varrho_{r k}\right)$

$\boldsymbol{\Sigma}_{k-1}=\left[\begin{array}{lll}\hat{\mathbf{x}}_{k-1} & \hat{\mathbf{x}}_{k-1}+\bar{\tau} \sqrt{\mathbf{P}_{k-1}} & \hat{\mathbf{x}}_{k-1}-\bar{\tau} \sqrt{\mathbf{P}_{k-1}}\end{array}\right]$

$\boldsymbol{\Sigma}_{k \mid k-1}=\mathcal{F}\left(\boldsymbol{\Sigma}_{k-1}, \mathbf{u}_{k-1}\right)$

$\boldsymbol{\Lambda}_{k \mid k-1}=\mathcal{G}\left(\boldsymbol{\Sigma}_{k \mid k-1}, \boldsymbol{\Sigma}_{k-1}\right)$

Optimal prediction of the mean, output, and covariance: $\hat{\mathbf{x}}_{m k}=\sum_{i=0}^{2 N} W_{i}^{m} \boldsymbol{\Sigma}_{i, k \mid k-1}, \hat{\mathbf{y}}_{m k}=\sum_{i=0}^{2 N} W_{i}^{m} \boldsymbol{\Lambda}_{i, k \mid k-1}$

$\mathbf{P}_{m k}=\sum_{i=0}^{2 N} W_{i}^{c}\left(\boldsymbol{\Sigma}_{i, k \mid k-1}-\hat{\mathbf{x}}_{m k}\right)\left(\boldsymbol{\Sigma}_{i, k \mid k-1}-\hat{\mathbf{x}}_{m k}\right)^{T}+\bar{Q}_{k}$

Modified covariance matrices:

$\mathbf{P}_{\tilde{\mathbf{x}}_{k} \tilde{\mathbf{y}}_{k}}=\sum_{i=0}^{2 N} W_{i}^{c}\left(\boldsymbol{\Sigma}_{i, k \mid k-1}-\hat{\mathbf{x}}_{m k}\right)\left(\boldsymbol{\Lambda}_{i, k \mid k-1}-\hat{\mathbf{y}}_{m k}\right)^{T}$

$\mathbf{P}_{\tilde{\mathbf{y}}_{k} \tilde{\mathbf{y}}_{k}}=\sum_{i=0}^{2 N} W_{i}^{c}\left(\boldsymbol{\Lambda}_{i, k \mid k-1}-\hat{\mathbf{y}}_{m k}\right)\left(\boldsymbol{\Lambda}_{i, k \mid k-1}-\hat{\mathbf{y}}_{m k}\right)^{T}+$ $\bar{R}_{k}$

State and covariance update:

$K_{k}=\mathbf{P}_{\tilde{\mathbf{x}}_{k} \tilde{\mathbf{y}}_{k}} \mathbf{P}_{\tilde{\mathbf{y}}_{k} \tilde{\mathbf{y}}_{k}}^{-1}$

$\hat{\mathbf{x}}_{k}=\hat{\mathbf{x}}_{m k}+K_{k}\left(\mathbf{y}_{k}-\hat{\mathbf{y}}_{m k}\right)$

$\mathbf{P}_{k}=\mathbf{P}_{m k}-K_{k} \mathbf{P}_{\tilde{\mathbf{y}}_{k} \tilde{\mathbf{y}}_{k}} K_{k}^{T}$

Optimality and convergence of the UKF state estimation method is discussed in [54], [56] and makes this method appropriate for such force estimation with road uncertainties and measurement noises.

The velocity estimator in the next section requires normalization of the longitudinal/lateral forces at each corner. Thus, estimated forces are normalized as

$$
\mu_{x_{i j}}=F_{x_{i j}} / F_{z_{i j}} \quad \mu_{y_{i j}}=F_{y_{i j}} / F_{z_{i j}}
$$

at each corner $i j$ using calculated vertical forces in the vehicle coordinate frame. Lateral force estimates from (10) is normalized by (13) to be implemented in the lateral velocity estimator (38).

\section{Vertical force calculation}

Estimated longitudinal/lateral forces in (2) and (10) should be normalized to be utilized in the velocity estimator robust to the road condition, which will be explored in the next section. Normal (vertical) forces at each axle/tire and longitudinal/lateral dynamics are schematically illustrated in Fig. 2b. The effects of the vehicle body's vertical motion and the roll/pitch angles are not commonly considered in the existing vertical force estimation methods [4], [5]. To tackle this issue, the vertical force estimator module is developed in this paper using vehicle dynamics and incorporation of the vehicle angles. The longitudinal and vertical acceleration components of the longitudinal dynamics are defined as follows in the body-fixed coordinates:

$$
\begin{aligned}
& \bar{a}_{\theta x}=a_{x} \cos \theta_{v}+a_{z} \sin \theta_{v} \\
& \bar{a}_{\theta z}=a_{z} \cos \theta_{v}-a_{x} \sin \theta_{v},
\end{aligned}
$$

where $a_{x}, a_{z}$ are the measured longitudinal and vertical accelerations by an IMU attached to the sprung mass, which are influenced by the kinematics of the vehicle's CG, vehicle pitch angle $\theta_{v}$, and the road grade angle $\theta_{r}$. Normal forces at front and rear axles, thus can be calculated by:

$$
\begin{aligned}
& F_{z_{f}}=-\frac{m}{L_{f}+L_{r}}\left(h_{C G} \bar{a}_{\theta x}-L_{r} \bar{a}_{\theta z}\right) \\
& F_{z_{r}}=\frac{m}{L_{f}+L_{r}}\left(h_{C G} \bar{a}_{\theta x}+L_{f} \bar{a}_{\theta z}\right),
\end{aligned}
$$

where the vehicle's body pitch is denoted by $\theta_{v}$, and $h_{C G}$ is the height of the vehicle's center of gravity. Similarly, the lateral and vertical acceleration components of the lateral dynamics in the body-fixed coordinates are defined by:

$$
\begin{gathered}
\bar{a}_{\phi y}=a_{y} \cos \phi_{v}+a_{z} \sin \phi_{v} \\
\bar{a}_{\phi z}=a_{z} \cos \phi_{v}-a_{y} \sin \phi_{v},
\end{gathered}
$$

in which $\phi_{v}$ is the vehicle's body roll angle and $a_{y}$ is the measured lateral acceleration by IMU which contains the kinematics of the vehicle's CG, vehicle roll $\phi_{v}$ angle, and the road bank angle $\phi_{r}$. Therefore, using (15) and defining equivalent masses at each axle $m_{i}=\frac{F_{z_{i}}}{g}, i \in\{f, r\}$ (front and rear axles), the normal forces at each corner becomes:

$$
\begin{aligned}
& F_{z_{i L}}=\frac{m_{i}}{T_{r_{i}}}\left[\bar{a}_{\phi z}\left(\frac{T_{r_{i}}}{2}-h_{r c} \sin \phi_{v}\right)-\bar{a}_{\phi y} h_{C G}\right] \\
& F_{z_{i R}}=\frac{m_{i}}{T_{r_{i}}}\left[\bar{a}_{\phi z}\left(\frac{T_{r_{i}}}{2}+h_{r c} \sin \phi_{v}\right)+\bar{a}_{\phi y} h_{C G}\right],
\end{aligned}
$$

in which $h_{r c}$ is the height of the roll center, $T_{r_{i}}$ represents the front and rear track widths (i.e. $T_{r_{f}}, T_{r_{r}}$ ), and left and right sides are denoted by $L, R$. 


\section{VELOCITY ESTIMATION}

This section presents a velocity estimation methodology robust to the road friction. As conferred in section I, because of difficulties in dealing with time-varying tire parameters and unknown road conditions, conventional kinematic-based velocity estimators employ acceleration measurement and rely on GPS data intermittently. Linear, Kalman-based, or nonlinear observers are used in such kinematic methods [15], [16], [19] without using a tire model. Solving the longitudinal $\dot{V}_{x}=a_{x}+r V_{y}+\vartheta_{x}$ and lateral $\dot{V}_{y}=a_{y}-r V_{x}+\vartheta_{y}$ kinematics with removing bias and noises $\vartheta_{x}, \vartheta_{y}$ from using GPS, the kinematic methods estimate the longitudinal and lateral velocities $V_{x}, V_{y}$. However, using an accurate GPS device and unavailability of reliable signals in many circumstances imposes extra costs and uncertainties for production vehicles. On the other hand, the performance of the velocity estimators based on tire forces is practically limited because of uncertain road friction and tire parameters, especially for saturation regions. Consequently, the kinematic approach is combined with the LuGre model's internal states at each corner in this section to tackle these issues.

The average lumped LuGre model [58] is utilized in this study to estimate velocities because of its accuracy and the dynamics on its internal states. This model symbolizes the distributed force over the patch line with some simplifications of normal force distribution. The internal state $\bar{z}_{q}$ for each direction $q \in\{x, y\}$ in the pure-slip model is described as:

$$
\begin{gathered}
\dot{\bar{z}}_{q}=v_{r q}-\left(\kappa_{q} R_{e}|\omega|+\frac{\sigma_{0 q}\left|v_{r q}\right|}{\theta g\left(v_{r q}\right)}\right) \bar{z}_{q}, \\
\mu_{q}=\sigma_{0 q} \bar{z}_{q}+\sigma_{1 q} \dot{\bar{z}}_{q}+\sigma_{2 q} v_{r q},
\end{gathered}
$$

in which $\sigma_{0 q}, \sigma_{1 q}, \sigma_{2 q}$ are the rubber stiffness, damping, and relative viscous damping in longitudinal/lateral directions, respectively. $R_{e}$ is the rolling radius and the normalized force of the averaged lumped pure-slip LuGre model for each direction is denoted by $\mu_{q}$. The force distribution along the patch line is represented by parameter $\kappa_{q}$ in the average lumped model and can be a function of time, a constant, or may be approximated by an asymmetric trapezoidal scheme. The suggested value for $\kappa_{q}$ in [58] is $\kappa_{q}=\frac{7}{6 L_{t}}$, where $L_{t}$ is the tire patch length. The function, $g\left(v_{r q}\right)$ in the pure slip model is defined for the longitudinal and lateral directions as $g\left(v_{r q}\right)=\mu_{c}+\left(\mu_{s}-\mu_{c}\right) e^{-\left|\frac{v_{r q}}{V_{s}}\right|^{\bar{\alpha}}}$, in which $\mu_{c}, \mu_{s}$ are the normalized Coulomb friction and static friction, respectively. The vehicle stability is analysed with the pure and combinedslip LuGre tire models in [59]. The Stribeck velocity $V_{s}$ shows the transition between these two friction states. The tire parameter $\bar{\alpha}=0.5$ is assumed for this study. The longitudinal/lateral relative velocities are defined by $v_{r x}=R_{e} \omega-v_{x t}$ and $v_{r y}=-v_{y t}$ respectively. These relative velocities at the tire coordinates of the LuGre model represent the slip ratio $\lambda=\frac{R_{e} \omega-v_{x t}}{\max \left\{R_{e} \omega, v_{x t}\right\}}$ and slip angle $\alpha=\tan ^{-1} \frac{v_{y t}}{v_{x t}}$ in the mostly used tire models such as Burckhardt [27] and Pacejka [31] models. The level of tire and road adhesion is represented by introducing the road classification factor $\theta$ which may vary between $0<\theta \leq 1$ according to dry, wet, and icy conditions. Chen and Wang [60] suggested a recursive least square (RLS) estimator and an adaptive control law with a parameter projection approach for identification of this road classification parameter. Identification of this factor is also addressed in [38] by a sliding mode observer for estimation of the maximum transmissible torque and wheel slip. The unknown road friction term $\frac{\sigma_{0 q}\left|v_{r q}\right|}{\theta g\left(v_{r q}\right)} \bar{z}_{q}$ and changes in the rolling radius are unknown, considered as uncertainty terms, and will be described in the following subsections.

\section{A. Longitudinal velocity estimation}

The LuGre model's internal states (18) can be written in the presence of uncertainty $\Omega_{z}(t)$ as follows at each corner (tire) for the longitudinal direction:

$$
\dot{\bar{z}}_{x}=v_{r x}-\kappa_{x} R_{e}|\omega| \bar{z}_{x}+\Omega_{z x} .
$$

Uncertainty $\Omega_{z x}$ is replaced with the road friction term $\frac{\sigma_{0 x}\left|v_{r x}\right|}{\theta g\left(v_{r x}\right)} \bar{z}_{x}$ and is bounded. Moreover, the derivative of the relative velocity is also corrupted due to the sensor noise and bias [61]:

$$
\dot{v}_{r x}=R_{e} \dot{\omega}-\dot{v}_{x t}+\Omega_{a x},
$$

in which $\dot{\omega}$ is the wheel's rotational acceleration and $\dot{v}_{x t}$ represents the longitudinal acceleration in the tire coordinate system. $\dot{v}_{x t}$ is obtained from first, transformation of the corrected acceleration $\bar{a}_{x}+r V_{y}$ from CG to corners where $\bar{a}_{x}$ from (11) only contains the kinematic part, then, projection of the corner's acceleration into the tire coordinates. The term $\Omega_{a x}$ shows the deviation of the measured relative acceleration $R_{e} \dot{\omega}-\dot{v}_{x t}$ from $\dot{v}_{r x}$ because of the sensor noises. Establishing these equations allow the development of an observer to incorporate both tire deflections (20) and relative velocities (21) concurrently. The general form of the system dynamics is given as follows:

$$
\begin{aligned}
{\left[\begin{array}{c}
\dot{\bar{z}}_{x} \\
\dot{v}_{r x}
\end{array}\right] } & =\left[\begin{array}{cc}
-\kappa_{x} R_{e} \omega & 1 \\
0 & 0
\end{array}\right]\left[\begin{array}{c}
\bar{z}_{x} \\
v_{r x}
\end{array}\right]+\left[\begin{array}{l}
0 \\
1
\end{array}\right]\left(R_{e} \dot{\omega}-\dot{v}_{x t}\right)+\boldsymbol{\Omega}_{x} \\
& =A_{x}(\omega) \mathbf{x}+B_{x} u_{x}+\mathbf{\Omega}_{x}
\end{aligned}
$$

in which $\boldsymbol{\Omega}_{x}=\left[\begin{array}{ll}\Omega_{z x} & \Omega_{a x}\end{array}\right]^{T}$ and states are $\mathbf{x}=\left[\begin{array}{ll}\bar{z}_{x} & v_{r x}\end{array}\right]^{T}$. Substituting $\dot{\bar{z}}_{x}$ from (20) into the normalized longitudinal force equation of the pure-slip LuGre model (19), one can rewrite the output equation as:

$$
\begin{aligned}
& \mu_{x}=\left[\begin{array}{ll}
\left(\sigma_{0 x}-\sigma_{1 x} \kappa_{x} R_{e} \omega\right) & \left.\left(\sigma_{1 x}+\sigma_{2 x}\right)\right] \mathbf{x}+\sigma_{1 x} \Omega_{z x}
\end{array}\right. \\
& =C_{x}(\omega) \mathbf{x}+\sigma_{1 x} \Omega_{z x} \text {. }
\end{aligned}
$$

Thereby, the estimated output can be written as $\hat{\mu}_{x}=C_{x}(\omega) \hat{\mathbf{x}}$. Using the modified longitudinal kinematics (22) the following observer is proposed for the velocity estimation:

$$
\dot{\hat{\mathbf{x}}}=A_{x}(\omega) \hat{\mathbf{x}}+B_{x} u_{x}+\mathbf{L}_{x}\left(\mu_{x}-\hat{\mu}_{x}\right),
$$

where $\mathbf{L}_{x}=\left[\begin{array}{ll}L_{1 x} & L_{2 x}\end{array}\right]^{T}$ is the observer gain matrix and $\mu_{x}$ represents the normalized longitudinal forces discussed in section III. Taking into account that the systems dynamic is time-varying with respect to the wheel speed, the suggested 
estimation method must be designed for the corresponding uncertain LPV system.

The bounded time-varying parameter is the wheel speed $\omega: \mathbb{R}_{>0} \rightarrow \mathcal{S}_{p}$ where $\mathcal{S}_{p}$ is the set of vertices of the parameter interval $\left[\omega_{l}, \omega_{u}\right]$ and the parameter varying state transition matrix is $A_{x}(\omega) \in \mathbb{R}^{2 \times 2}$. The error dynamics $\dot{\mathbf{e}}_{x}=\dot{\mathbf{x}}-\dot{\hat{\mathbf{x}}}$ from (22) and (24) yields:

$$
\begin{aligned}
\dot{\mathbf{e}}_{x} & =\left(A_{x}(\omega)-\mathbf{L}_{x} C_{x}\right) \mathbf{e}_{x}-\mathbf{L}_{x} \sigma_{1 x} \Omega_{z x}+\mathbf{\Omega}_{x} \\
& =A_{e_{x}}(\omega) \mathbf{e}_{x}+\underbrace{\left[\begin{array}{cc}
1-L_{1 x} \sigma_{1 x} & 0 \\
-L_{2 x} \sigma_{1 x} & 1
\end{array}\right]}_{B_{e_{x}}} \boldsymbol{\Omega}_{x},
\end{aligned}
$$

1) Estimator's stability analysis: The objective of this subsection is to show that the error dynamics (25) is affinely quadratically stable over all possible trajectories of $\omega$. The state matrix $A_{e_{x}}(\omega)$ is said to be affinely dependent on the parameter $\omega$ when known and fixed matrices $A_{0 x}$ and $A_{1 x}$ exist such that $A_{e_{x}}(\omega)=A_{0 x}+\omega A_{1 x}$. The error dynamic matrix for both lateral and longitudinal directions $q \in\{x, y\}$ is introduced as:

$$
\begin{aligned}
A_{e_{q}} & =\left[\begin{array}{ll}
-L_{1 q} \sigma_{0 q} & -L_{1 q} p_{1 q} \\
-L_{2 q} \sigma_{0 q} & -L_{2 q} p_{1 q}
\end{array}\right]+\omega\left[\begin{array}{cc}
\kappa_{q} R_{e} p_{2 q} & 0 \\
\kappa_{q} R_{e} L_{2 q} \sigma_{1 q} & 0
\end{array}\right] \\
& =A_{0 q}+\omega A_{1 q}
\end{aligned}
$$

where $p_{1 q}=\sigma_{1 q}+\sigma_{2 q}$ and $p_{2 q}=L_{1 q} \sigma_{1 q}-1$. The bounded time-varying parameter and its time derivatives are in the sets $\omega_{p} \in\left[\omega_{l}, \omega_{u}\right]$ and $\dot{\omega}_{p} \in\left[\dot{\omega}_{l}, \dot{\omega}_{u}\right]$, respectively.

A linear system like (25) is affinely quadratically stable over all possible trajectories of the parameter vector $\omega(t)$ if $A_{e_{x}}\left(\omega_{m}\right)$ is stable $\left(\omega_{m}\right.$ is the average value of $\omega$ over the parameter span) and there exists an affine positive definite Lyapunov function

$$
V\left(e_{x}, \omega\right)=e_{x}^{T} P(\omega) e_{x}
$$

with $P(\omega)=P_{0}+\omega P_{1}>0$ such that $d V(\omega, \dot{\omega}) / d t<0$ for all initial conditions $\mathbf{x}_{0}$ and the additional multi-convexity constraint $A_{1 x}^{T} P_{1}+P_{1} A_{1 x} \geq 0$ holds [62]. The condition $\dot{V}<$ 0 resembles

$$
A_{e_{x}}^{T}\left(\omega_{p}\right) P\left(\omega_{p}\right)+P\left(\omega_{p}\right) A_{e_{x}}\left(\omega_{p}\right)+P\left(\dot{\omega}_{p}\right)-P_{0}<0,
$$

for all $(\omega, \dot{\omega}) \in \mathcal{S}_{p} \times \mathcal{S}_{r}$ where $\mathcal{S}_{r}$ is the set of corners of the rate in $\left[\dot{\omega}_{l}, \dot{\omega}_{u}\right]$. The affine quadratic stability condition implements the variation rate $\dot{\omega}(t)$, which makes it less conservative than the quadratic stability criteria. The error dynamics is affinely quadratically stable for the two sets of observer gains $L_{1 x} \in[0.5,0.9], L_{2 x} \in[60,210]$, obtained by several simulations and experimental tests on different road conditions and the vehicle parameters listed in Table I. Moreover, a rigorous method for determining the LPV observer gains is provided in the next subsection.

Stability of the system (25) will be guaranteed with the substitution of the operating regions $|\omega| \leq 180[\mathrm{rad} / \mathrm{s}]$ and $|\dot{\omega}| \leq 800\left[\mathrm{rad} / \mathrm{s}^{2}\right]$, which is practical for this case according to the sampling frequency $200[\mathrm{~Hz}]$ i.e. $T_{s}=0.005[\mathrm{~s}]$
TABLE I: Vehicle Spec. for Simulation \& Experiments

\begin{tabular}{cccc}
\hline Parameter & Unit & Value & Description \\
\hline \hline$m$ & {$[\mathrm{~kg}]$} & 2270 & Vehicle mass \\
$I_{z}$ & {$\left[\mathrm{~kg} \cdot \mathrm{m}^{2}\right]$} & 4650 & Vehicle moment of inertia \\
$I_{w}$ & {$\left[\mathrm{~kg} \cdot \mathrm{m}^{2}\right]$} & 1.68 & Wheel moment of inertia \\
$L_{w b}$ & {$[\mathrm{~m}]$} & 2.85 & Vehicle wheel base \\
$L_{f}, L_{r}$ & {$[\mathrm{~m}]$} & $1.42,1.43$ & Front \& rear axles to CG \\
$R_{e}$ & {$[\mathrm{~m}]$} & 0.33 & Effective radius \\
$h_{C G}$ & {$[\mathrm{~m}]$} & 0.65 & CG height \\
$h_{r c}$ & {$[\mathrm{~m}]$} & 0.54 & Roll axis height \\
$T_{r_{f}}, T_{r_{r}}$ & {$[\mathrm{~m}]$} & $1.62,1.56$ & Front and rear track width \\
\hline
\end{tabular}

and measurement errors in the wheel speed. Given the vehicle parameters listed in Table I, the tire rubber stiffness $\sigma_{0 x}=632.1[1 / \mathrm{m}]$, rubber damping $\sigma_{1 x}=0.76[\mathrm{~s} / \mathrm{m}]$, relative viscous damping $\sigma_{2 x}=0.0016[\mathrm{~s} / \mathrm{m}]$, load distribution factor $\kappa_{x}=8.32$, observer gains $L_{1 x}=0.68, L_{2 x}=183.1$ and the affinely dependent form of (26), considering a polytope with bounds on $\dot{\omega}$ to solve (28) yields the following numeric values for the symmetric matrix $P(\omega)$ :

$$
P(\omega)=\left[\begin{array}{cc}
1.2176-0.0011 \omega & 115.35-0.2227 \omega \\
115.35-0.2227 \omega & 80379.0-67.969 \omega
\end{array}\right] .
$$

2) Estimator's performance analysis: The objective is to find the observer gains such that the ratio of the estimation error to the disturbance energy is minimized considering the fact that the process disturbance $\Omega_{z x}$ and the measurement disturbance $\Omega_{a x}$ are bounded. Given a compact set $\omega \in\left[\omega_{l}, \omega_{u}\right]$ and a bounded rate of variation of $|\dot{\omega}|<\zeta_{\omega}$, for some $\zeta_{\omega}>0$ the system (25) is robustly exponentially stable if there exist a continuously differentiable positive definite matrix $P(\omega)$ and a matrix $\chi(\omega)$ such that the following LMI holds:

$$
\left[\begin{array}{ccc}
\varphi(\omega) & P(\omega)+\chi(\omega) B_{e 1} & C_{e}^{T} \\
* & -\gamma I & 0 \\
* & * & -\gamma I
\end{array}\right] \prec 0,
$$

where the symmetric terms are denoted by $*$ and $\varphi$ is:

$$
\begin{aligned}
\varphi(\omega) & =\left[A_{x}^{T}(\omega)-C_{x}^{T}(\omega) \mathbf{L}_{x}^{T}\right] P(\omega) \\
& +P(\omega)\left[A_{x}(\omega)-\mathbf{L}_{x} C_{x}(\omega)\right]+\frac{\partial P}{\partial \omega} \dot{\omega} .
\end{aligned}
$$

In order to isolate the observer gain effect, $B_{e_{x}}$ can be written as $B_{e_{x}}=I_{2 \times 2}+\mathbf{L}_{x} B_{e 1}$, in which $B_{e 1}=\left[\begin{array}{ll}\sigma_{1 x} & 0\end{array}\right]$. The induced $\mathcal{L}_{2}$ norm from the input disturbance to the output error is less than the performance level $\gamma>0$. The LMI (30) is obtained by taking derivative of the Lyapunov function $V\left(e_{x}, \omega\right)=e_{x}^{T} P(\omega) e_{x}$, imposing the condition $\varphi(\omega)<0$, and using the Bounded Real Lemma. Employing $\chi(\omega)=P(\omega) \mathbf{L}_{x}$, one can rewrite:

$$
\varphi(\omega)=A_{x}^{T} P+P A_{x}-\chi C_{x}-C_{x}^{T} \chi^{T}+\frac{\partial P}{\partial \omega} \dot{\omega}
$$

The LMI (30) guarantees that $\dot{V}+\mathbf{e}_{x}^{T} \mathbf{e}_{x}-\gamma^{2} \boldsymbol{\Omega}_{x}^{T} \boldsymbol{\Omega}_{x}<0$. The set of gains will be calculated by $\mathbf{L}_{x}=P(\omega)^{-1} \chi(\omega)$. The infinite dimensional parameter-varying LMI (30) with $\varphi(\omega)$ from (32) can explicitly be expressed in a finite dimensional 
problem with the parametric matrices and using appropriate basis functions. The positive definite matrix $P$ and matrix $\varphi$ are defined as $P(\omega):=\sum_{i=0}^{f} P_{i} \omega^{i}$ and $\varphi(\omega):=\sum_{i=0}^{f} \varphi_{i} \omega^{i}$ respectively and the set $\omega=\left[\begin{array}{ll}0 & 140\end{array}\right]$ is gridded to $\mathcal{N}_{g r}^{i=0}=140$ points. The time-varying observer gains $L_{1}, L_{2}$ are depicted in Fig. 3 for the longitudinal observer and the vehicle parameters provided in Table I. The YALMIP package [63] is integrated with MOSEK to solve the LMIs.
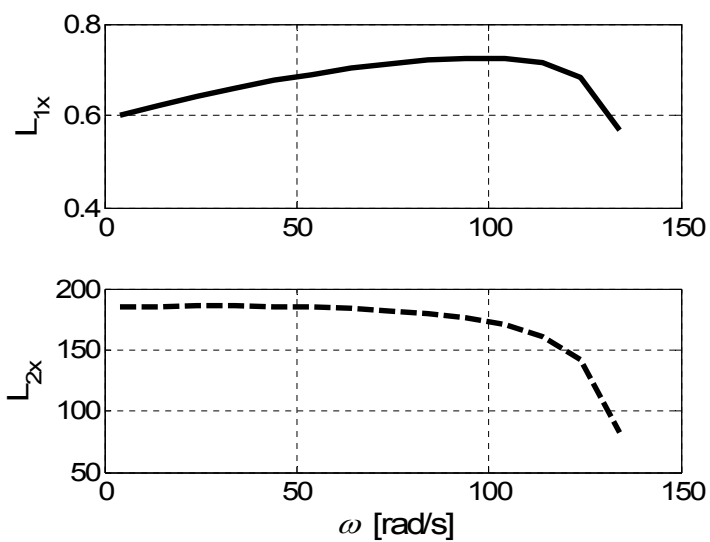

Fig. 3: Time-varying observer gains for the longitudinal estimator

The estimated relative longitudinal velocities $\hat{v}_{r x_{i j}}$ at each corner from (24) are used for the longitudinal velocity estimation at the tire coordinates as $\hat{v}_{x t_{i j}}=R_{e} \omega_{i j}-\hat{v}_{r x_{i j}}$. Afterward, each corner's longitudinal velocity in the vehicle coordinates $\hat{v}_{x_{i j}}$ yields:

$$
\hat{v}_{x_{i j}}=\hat{v}_{x t_{i j}} \cos \delta-\hat{v}_{y t_{i j}} \sin \delta
$$

in which $\delta$ is the steering angle at corners and the estimated lateral velocity at each corner's tire coordinates is denoted by $\hat{v}_{y t_{i j}}$. The corner-based lateral velocity estimation will be described in the next subsection.

To tackle the high slip conditions and provide a smooth estimation with removing outliers, weighted estimated longitudinal velocities at each corner are used for the estimation of the vehicle speed, $\hat{V}_{x}$. Specifically, each axles' longitudinal velocities are defined by $\hat{v}_{x_{f}}$ and $\hat{v}_{x_{r}}$ that are the mean values between $\hat{v}_{x_{f L}}, \hat{v}_{x_{f R}}$ for the front axle and $\hat{v}_{x_{r L}}, \hat{v}_{x_{r R}}$ for the rear axle respectively. Then, the longitudinal velocity of the vehicle $\hat{V}_{x}$ at $\mathrm{CG}$ is achievable by adaptive weighted velocity mapping method, which is allocating adaptive weights $\mathcal{W}_{x}^{f}, \mathcal{W}_{x}^{r}$ to each axle as $\hat{V}_{x}=\mathcal{W}^{f} \hat{v}_{x_{f}}+\mathcal{W}^{r} \hat{v}_{x_{r}}$. Adaptive weights are defined with respect to the maximum slip ratio of each axle as functions:

$$
\mathcal{W}_{x}^{i}=\mathcal{W}_{s_{x}}+\overline{\mathcal{W}}_{x} \tan ^{-1}\left[\rho_{\mathrm{w}_{x}}\left(\lambda_{a_{m}}-\lambda_{\mathrm{w}_{t h}}\right)\right],
$$

and $\mathcal{W}_{x}^{-i}=1-\mathcal{W}_{x}^{i}$ where $i \in\{f, r\}$ and $-i$ represents another axle i.e. $-i \in\{r, f\}$. The weight range coefficient is $\overline{\mathcal{W}}_{x}=\left(\overline{\mathcal{W}}_{u b_{x}}-\overline{\mathcal{W}}_{l b_{x}}\right) / \pi$ where upper and lower bounds on the allocated weights are expressed by $\overline{\mathcal{W}}_{u b_{x}}, \overline{\mathcal{W}}_{l b_{x}}$. The slip ratio threshold at which the weight of each axle is the same is denoted by $\lambda_{\mathrm{w}_{t h}}$. The maximum slip ratio of axles is used to allocate a smaller weight to an axle with higher slip ratio; it is achievable by

$$
\lambda_{a_{m}}=\max \left\{\Sigma_{i}^{x}, \Sigma_{-i}^{x}\right\}
$$

where $\Sigma_{i}^{x}=\left|\lambda_{i L}\right|+\left|\lambda_{i R}\right|$ is defined for an axle and $\Sigma_{-i}^{x}=$ $\left|\lambda_{-i L}\right|+\left|\lambda_{-i R}\right|$ is written for another axle. The shape of the axle's weight function can change with the parameters $\rho_{\mathrm{w}_{x}}$ and the static weight $\mathcal{W}_{s_{x}}$. Afterward, the calculated velocity at $\mathrm{CG}$ is remapped again to each corner to have slip ratios for the stability and traction control systems.

\section{B. Lateral velocity estimation}

The LuGre output equation for the lateral direction can be described as follows with states $\mathbf{x}_{l}=\left[\begin{array}{ll}\bar{z}_{y} & v_{r y}\end{array}\right]^{T}$ :

$$
\begin{aligned}
\mu_{y} & =\left[\begin{array}{ll}
\left(\sigma_{0 y}-\sigma_{1 y} \kappa_{y} R_{e} \omega\right) & \left(\sigma_{1 y}+\sigma_{2 y}\right)
\end{array}\right] \mathbf{x}_{l}+\sigma_{1 y} \Omega_{z y} \\
& =C_{y}(\omega) \mathbf{x}_{l}+\sigma_{1 y} \Omega_{z y} .
\end{aligned}
$$

Employing the lateral LuGre internal state from (18) and the relative lateral acceleration $\dot{v}_{r y}=-\dot{v}_{y t}+\Omega_{a y}$ with the projected lateral acceleration $\dot{v}_{y t}$ in the tire coordinate system, the newly proposed lateral dynamics can be developed. $\dot{v}_{y t}$ is obtained from first, transformation of the corrected acceleration $\bar{a}_{y}-r V_{x}$ from CG to corners where $\bar{a}_{y}$ from (11) only includes the kinematic part, then, projection of the corner's acceleration into the tire coordinates.

Therefore, (22) can be rewritten for the lateral direction as:

$$
\dot{\mathbf{x}}_{l}=A_{y}(\omega) \mathbf{x}_{l}+B_{y} u_{y}+\boldsymbol{\Omega}_{y} .
$$

using state and input matrices similar to the longitudinal case $A_{y}=\left[\begin{array}{lll}-\kappa_{y} R_{e} \omega & 1 ; 0 & 0\end{array}\right], B_{y}=B_{x}$ and $u_{y}=-\dot{v}_{y t}$. Uncertainties in the lateral states are denoted by $\Omega_{y}=\left[\begin{array}{ll}\Omega_{z y} & \Omega_{a y}\end{array}\right]^{T}$. The state estimator can be expressed as follows for the lateral direction:

$$
\dot{\hat{\mathbf{x}}}_{l}=A_{y}(\omega) \hat{\mathbf{x}}_{l}+B_{y} u_{y}+\mathbf{L}_{y}\left(\mu_{y}-\hat{\mu}_{y}\right),
$$

in which $\mathbf{L}_{y}=\left[\begin{array}{ll}L_{1 y} & L_{2 y}\end{array}\right]^{T}$. The error dynamics is then developed as:

$$
\dot{\mathbf{e}}_{y}=A_{e_{y}}(\omega) \mathbf{e}_{y}+\underbrace{\left[\begin{array}{cc}
1-L_{1 y} \sigma_{1 y} & 0 \\
-L_{2 y} \sigma_{1 y} & 1
\end{array}\right]}_{B_{e_{y}}} \boldsymbol{\Omega}_{y},
$$

where $A_{e_{y}}=\left(A_{y}-\mathbf{L}_{y} C_{y}\right)$. The error dynamics (39) for the proposed lateral velocity estimator represents a linear parameter-varying system and its stability can be investigated using the affine quadratic stability criteria discussed in the previous subsection.

Remark 2: The error dynamics (39) is affinely quadratically stable over all possible trajectories of $\omega$. Analogous to the longitudinal case, the state matrix $A_{e_{y}}(\omega)$ can be written in the affine form $A_{e_{y}}(\omega)=A_{0 y}+\omega A_{l y}$ with the fixed matrices $A_{0 y}$ and $A_{1 y}$ from (26). $A_{e_{y}}\left(\omega_{m}\right)$ is stable and there exists an affine positive definite Lyapunov function $V\left(e_{y}, \omega\right)=e_{y}^{T} P(\omega) e_{y}$ with $P(\omega)=P_{0}+\omega P_{1}>0$ such that $d V(\omega, \dot{\omega}) / d t<0$ 
for all initial conditions $\mathbf{x}_{l_{0}}$ and the additional multi-convexity constraint $A_{1 y}^{T} P_{1}+P_{1} A_{1 y} \geq 0$ holds. The condition $\dot{V}<0$ resembles

$$
A_{e_{y}}^{T}\left(\omega_{p}\right) P\left(\omega_{p}\right)+P\left(\omega_{p}\right) A_{e_{y}}\left(\omega_{p}\right)+P\left(\dot{\omega}_{p}\right)-P_{0}<0
$$

The error dynamics is affinely quadratically stable for the two sets of observer gains $L_{1 y} \in[0.8,1.3], L_{2 y} \in[6,19]$, obtained by several road experiments. Given the tire specifications $\sigma_{0 y}=181.5[1 / m], \sigma_{1 y}=0.81[s / m], \sigma_{2 y}=0.001[\mathrm{~s} / \mathrm{m}]$, $\kappa_{y}=12.84$, observer gains $L_{1 y}=1.11, L_{2 y}=16.7$, the vehicle parameters in Table I, and the affinely dependent form of (26), solving (40) with considering a polytope and bounded wheel acceleration $|\dot{\omega}| \leq 800\left[\mathrm{rad} / \mathrm{s}^{2}\right]$ yields:

$$
P(\omega)=\left[\begin{array}{cc}
4.7718-0.0070 \omega & 154.87+0.0038 \omega \\
154.87+0.0038 \omega & 75627.0-0.0013 \omega
\end{array}\right]
$$

Similar to the longitudinal case, parameter-varying observer gains are obtained using LMI (30) for the lateral direction and the outcomes are illustrated in Fig. 4.
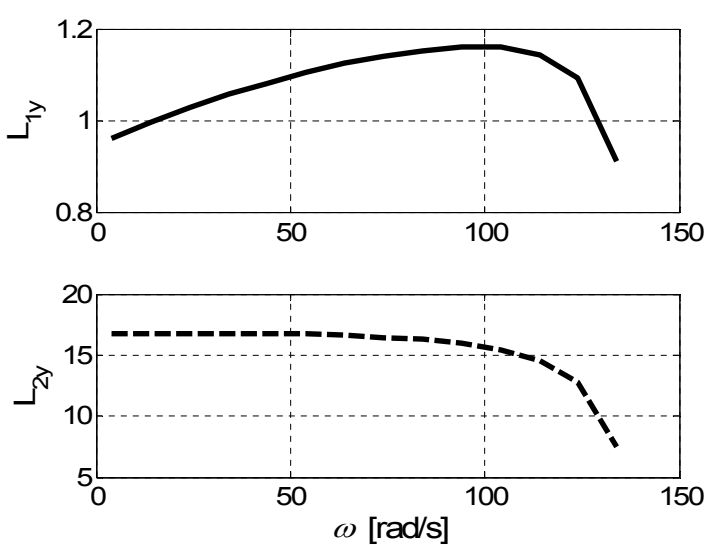

Fig. 4: Lateral observer's time-varying gains

Analogous to the longitudinal case, each corner's lateral velocity $\hat{v}_{y_{i j}}$ in the body-fixed vehicle coordinates is achievable from:

$$
\hat{v}_{y_{i j}}=\hat{v}_{x t_{i j}} \sin \delta+\hat{v}_{y t_{i j}} \cos \delta,
$$

where $\hat{v}_{y t_{i j}}$ and $\hat{v}_{x t_{i j}}$ are the estimated lateral and longitudinal velocities at the tire coordinates respectively. The lateral velocity in the tire coordinates is $\hat{v}_{y t_{i j}}=-\hat{v}_{r y_{i j}}$ and the relative velocity $\hat{v}_{r y_{i j}}$ is obtained from (38) at each corner.

The lateral velocities at each corner $\hat{v}_{y_{i j}}$ are then utilized for definition of the front and rear axle's lateral velocities $\hat{v}_{y_{f}}, \hat{v}_{y_{r}}$ respectively. Each axle's lateral velocities are obtained by $\hat{v}_{y_{f}}=-r L_{f}+\left(\hat{v}_{y_{f L}}+\hat{v}_{y_{f R}}\right) / 2$ for the front axle and $\hat{v}_{y_{r}}=r L_{r}+\left(\hat{v}_{y_{r L}}+\hat{v}_{y_{r R}}\right) / 2$ for the rear axle. Similar to the longitudinal case, using weighted estimated axles' lateral velocities, the vehicle lateral velocity $\hat{V}_{y}$ at the $\mathrm{CG}$ is expressed as follows:

$$
\hat{V}_{y}=\mathcal{W}_{y}^{f} \hat{v}_{y_{f}}+\mathcal{W}_{y}^{r} \hat{v}_{y_{r}}
$$

where $\mathcal{W}_{y}^{f}$ and $\mathcal{W}_{y}^{r}$ are adaptive weights for each axle and are defined similar to the longitudinal case (34), but with respect to the maximum slip angle at each axle as in

$$
\mathcal{W}_{y}^{i}=\mathcal{W}_{s_{y}}+\overline{\mathcal{W}}_{y} \tan ^{-1}\left[\rho_{\mathrm{w}_{y}}\left(\alpha_{a_{m}}-\alpha_{\mathrm{w}_{t h}}\right)\right]
$$

with the slip angles threshold $\alpha_{\mathrm{w}_{t h}}$. The weight range coefficient for the lateral direction is denoted by $\overline{\mathcal{W}}_{y}=\left(\overline{\mathcal{W}}_{u b_{y}}\right.$ $\left.\overline{\mathcal{W}}_{l b_{y}}\right) / \pi$ with the upper and lower bounds $\overline{\mathcal{W}}_{u b_{y}}, \overline{\mathcal{W}}_{l b_{y}}$. Parameters $\rho_{\mathrm{w}_{y}}$ and $\mathcal{W}_{s_{y}}$ are introduced to change the shape of the lateral axle's weight function. To address the high slip angle scenarios and provide smooth estimation, the maximum slip angle of axles $\alpha_{a_{m}}=\max \left\{\Sigma_{i}^{y}, \Sigma_{-i}^{y}\right\}$ are utilized to allocate a smaller weight to an axle with higher slip angle. Each axle's slip angle is defined by

$$
\Sigma_{i}^{y}=\left|\alpha_{i L}\right|+\left|\alpha_{i R}\right|, \quad \Sigma_{-i}^{y}=\left|\alpha_{-i L}\right|+\left|\alpha_{-i R}\right| .
$$

Experimental results of the developed force and velocity estimators with weighted axles' velocity scheme are provided in the next section.

\section{RESULTS AND DISCUSSION}

This section includes experimental tests for validation of the longitudinal and lateral state estimators on an instrumented SUV with the specifications given in Table I. The test platform is a four-wheel-independent-drive electric vehicle.

The RT2500 6-axis GPS and IMU system and the Tire Force/Moment Measurement module are used for validation of the longitudinal, lateral, and vertical force estimators. The Controller \& Estimator module requires longitudinal and lateral accelerations, yaw rate, wheel speed as well as the wheel torques, which are measured using an IMU, regular ABS wheel speed sensors, and electric actuators respectively as shown in Fig. 5. Measured signals are communicated using a CAN-bus. Real-time acquisition and processing of sensory information and the developed algorithm is done using the dSPACE $R$ MicroAutobox. The dSPACE compiles measurements for MATLAB/SIMULINK, and the controller provides control signals for the dSPACE as well. Visualization of the experiment results is performed through the ControlDesk. The sampling frequency for the experiment is set to be $200[\mathrm{~Hz}]$.

First, road experiments using a four independent wheel drive SUV are used in this section to evaluate the force estimation methods, then the performance of the proposed velocity estimator is studied. To validate the estimated forces, this vehicle is equipped with additional sensors for direct measurement of tire forces and moments at each corner.

\section{A. Force estimation results}

In order to assess the proposed approach in combined-slip conditions, in which the tire capacities are reduced due to high slip ratio as well the high slip angles in each longitudinal/lateral direction, a harsh lane change (LC) maneuver is performed. Experimental results of the force estimation at the front-right wheel in a harsh LC maneuver on the wet road are demonstrated in Fig. 6a and compared with the measurement. The performed combined-slip maneuver is harsh, but the 


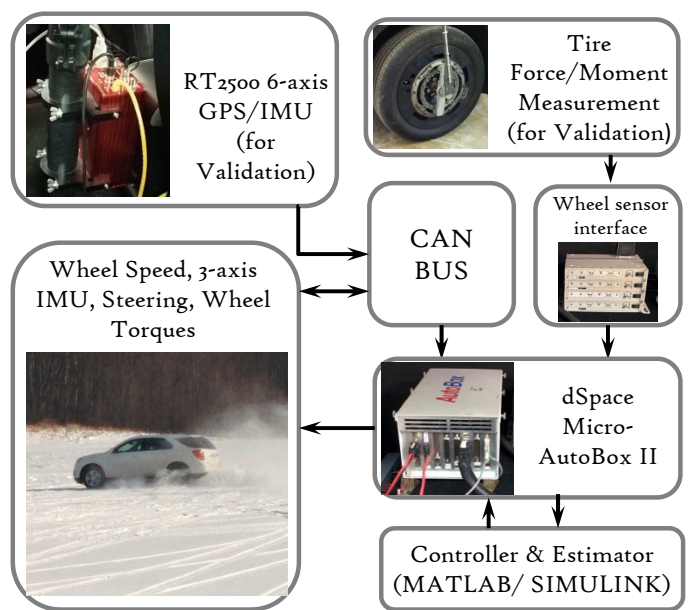

Fig. 5: The I/O and hardware layout

suggested observer handles the oscillations in the transient regions. A brake-in-turn (BiT) maneuver accompanied by hard acceleration on the packed snow (with $\mu \approx 0.3$ ) is also done and the force estimation approach is validated by the filtered measurements in Fig. $6 \mathrm{~b}$ at the front-right wheel.
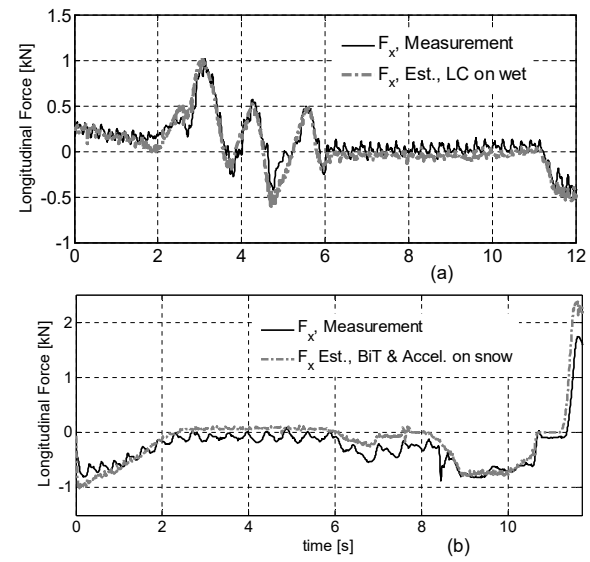

Fig. 6: Longitudinal forces for (a) harsh steering and LC on wet road $\mu \approx 0.5$ (b) BiT and acceleration on snow

The vehicle speed in harsh LC with brake on wet road (i.e. Fig. 6a) changed from $10.9[\mathrm{~m} / \mathrm{s}]$ to $9.6[\mathrm{~m} / \mathrm{s}]$ at $t=11.3[\mathrm{~s}]$ then decreased to $V_{x}=7.3[\mathrm{~m} / \mathrm{s}]$ at the end. In the BiT maneuver on packed snow (i.e. Fig. 6b), vehicle started from $V_{x}=8.4[\mathrm{~m} / \mathrm{s}]$ and stopped at $t=10.5[\mathrm{~s}]$, then accelerated to $3.1[\mathrm{~m} / \mathrm{s}]$ at the end of the experiment. The fluctuations observed in the filtered force profile measurement are attributed to the low-stick characteristics of the packed snow. Uncertainties in the effective radius and wheel speed derivative are tackled by tuning the observer gains $\eta_{1}, \eta_{3}$ and the observer provides smooth outcomes. The selected gains for these road experiments are $\eta_{1}=1.8, \eta_{2}=11.4$ and $\eta_{3}=50.6$.
Performance of the lateral and vertical force estimators on dry and slippery surfaces is examined in several road experiments with the process and measurement noise covariance matrices $\bar{Q}=0.13^{2} I_{3 \times 3}, \bar{R}=0.012^{2} I_{3 \times 3}$ for the lateral case. Results of the proposed force estimator in a lane change on the dry road is presented in Fig. 7 and compared with the measurement. The vehicle speed is $V_{x}=12[\mathrm{~m} / \mathrm{s}]$ at the beginning of the maneuver.
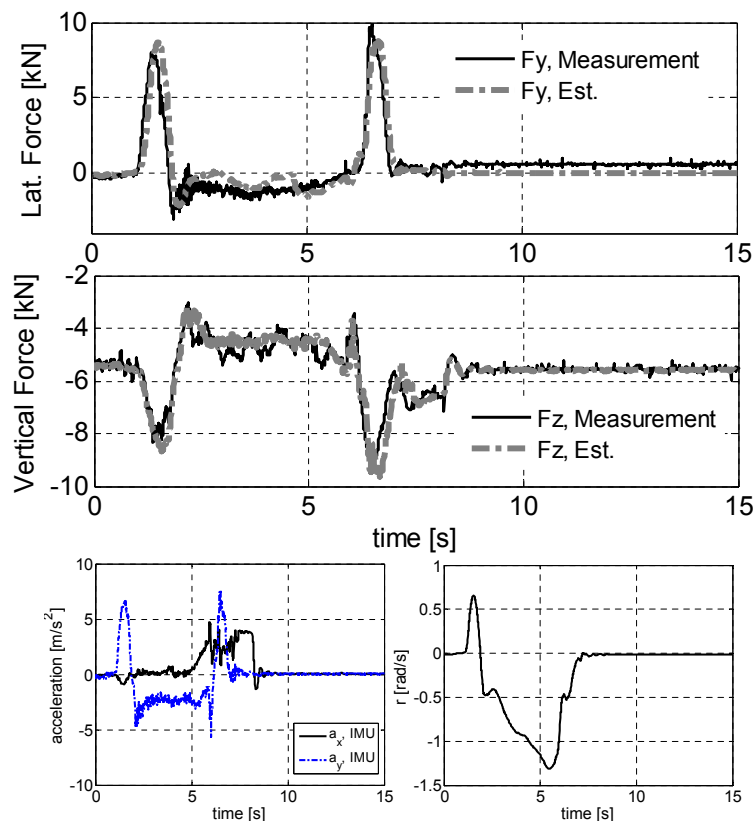

Fig. 7: Lateral force estimation, LC on a dry surface

The measured accelerations and yaw rate are also provided to show the characteristics of the test. Accuracy of the developed estimators are evaluated in different maneuvers with the normalized root mean square of NRMS the error defined by:

$$
\varepsilon_{n}=\frac{\sqrt{\sum_{i=1}^{N_{s}}\left(\hat{s}_{i}-s_{i}\right)^{2} / N_{s}}}{s_{m}},
$$

where the estimated and measured signals are denoted by $s$ and $\hat{s}$ respectively, $N_{s}$ is the number of collected signal samples during a driving scenario (DLC, BiT, LC etc.), and $s_{m}=$ $\max _{i=1 \ldots N_{s}}\left|s_{i}\right|$ shows the maximum value of the measured signal.

A harsh steering on an icy road is done and the results of the front left corner are illustrated for the All-Wheel-Drive (AWD) case in Fig. 8. The maneuver ended up on a surface with packed snow which is highly slippery itself with $\mu \approx 0.3$. The vehicle longitudinal velocity is $6.1 \leq V_{x} \leq 7.7[\mathrm{~m} / \mathrm{s}]$ for this test.

In summary, Table II provides the maximum and NRMS of the longitudinal, lateral, and vertical forces in different driving scenarios and on various road frictions.

Table II substantiates that the NRMS of the estimated longitudinal forces is less than $7.4 \%$ for the performed maneuvers on dry, wet, and snowy roads. This normalized error 

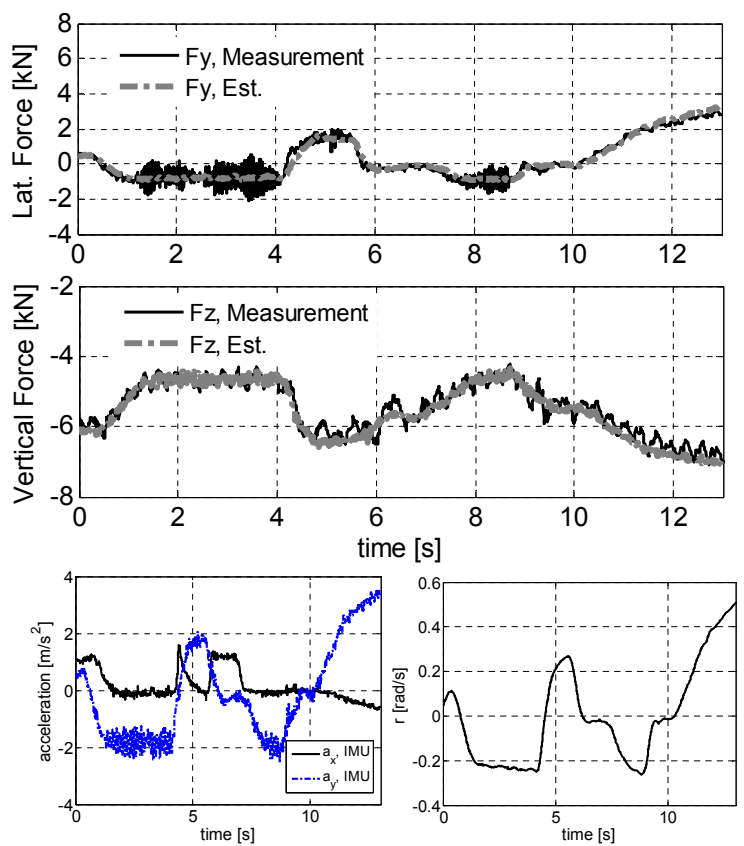

Fig. 8: Force estimates, steering on ice then packed snow

TABLE II: NRMS of the Errors for the Longitudinal, Lateral, and Vertical Force Estimators at each Corner (wheels)

\begin{tabular}{l|cccccc}
\hline \multirow{2}{*}{$\begin{array}{l}\text { Estimated } \\
\text { forces }\end{array}$} & \multicolumn{2}{|c}{ DLC on dry } & \multicolumn{2}{c}{ LC on wet } & \multicolumn{2}{c}{ BiT/Accel., snow } \\
\cline { 2 - 7 } & $\varepsilon_{n}[\%]$ & $s_{m}[N]$ & $\varepsilon_{n}[\%]$ & $s_{m}[N]$ & $\varepsilon_{n}[\%]$ & $s_{m}[N]$ \\
\hline \hline$F_{x_{f L}}$ & 4.31 & 4751 & 5.89 & 4569 & 5.43 & 2056 \\
$F_{x_{f R}}$ & 3.90 & 4042 & 6.12 & 4571 & 4.57 & 2051 \\
$F_{x_{r L}}$ & 4.72 & 3996 & 4.33 & 4574 & 6.59 & 747 \\
$F_{x_{r R}}$ & 4.02 & 3991 & 5.95 & 4577 & 7.38 & 740 \\
\hline$F_{y_{f L}}$ & 2.94 & $1.01 e+4$ & 6.03 & 6380 & 5.56 & 3185 \\
$F_{y_{f R}}$ & 3.19 & 9989 & 5.11 & 6548 & 6.82 & 1196 \\
$F_{y_{r L}}$ & 3.86 & 9208 & 5.24 & 4089 & 6.17 & 2749 \\
$F_{y_{r R}}$ & 2.73 & 8537 & 4.65 & 4180 & 7.08 & 1420 \\
\hline$F_{z_{f L}}$ & 2.06 & $1.29 e+4$ & 3.20 & $1.06 e+4$ & 4.01 & 8673 \\
$F_{z_{f R}}$ & 3.44 & $1.23 e+4$ & 2.93 & $1.05 e+4$ & 2.80 & 6482 \\
$F_{z_{r L}}$ & 1.91 & $1.07 e+4$ & 2.68 & 8110 & 3.19 & 7106 \\
$F_{z_{r R}}$ & 2.24 & $1.06 e+4$ & 3.47 & 8231 & 2.36 & 6297 \\
\hline
\end{tabular}

is $\varepsilon_{n} \leq 7.1 \%$ for the lateral forces and $\varepsilon_{n} \leq 4 \%$ for the vertical forces respectively. These normalized $R M S$ of the errors confirm effectiveness of the algorithm for the corner-based force estimation on dry and slippery roads. Observed errors between the measured and estimated forces in Table II for the force estimators may have several sources such as camber angle, which has not been modeled in the estimation algorithm. Moreover, inaccurate inertial parameters and uncertainties in the CG location contribute to such errors.

\section{B. Velocity estimation results}

So far, outcomes of the force estimators are investigated. In the following, several driving scenarios such as launch on ice, lane change (LC) with harsh steering, and acceleration-inturn (AiT) are examined on various roads and experimental results of the proposed velocity estimators (24), (38) are presented. For the road experiments, the rubber stiffness for the longitudinal and lateral directions are $\sigma_{0 x}=632.1[1 / \mathrm{m}]$ and $\sigma_{0 y}=181.5[1 / \mathrm{m}]$, the rubber damping is assumed as $\sigma_{1 x}=0.76, \sigma_{1 y}=0.81[\mathrm{~s} / \mathrm{m}]$, relative viscous damping is $\sigma_{2 x}=0.0016, \sigma_{2 y}=0.001[\mathrm{~s} / \mathrm{m}]$, and load distribution factor is $\kappa_{x}=8.32, \kappa_{y}=12.84$ for the velocity estimators.

1) Launch on split- $\mu$ : Longitudinal velocity estimator is examined in a harsh launch maneuver on a surface, which have two different friction conditions on the left and right sides and the results are shown in Fig. 9. The right wheels are on an icy road with $\mu \approx 0.2$, the left wheels are on a dry road, and the powertrain configuration is $A W D$.

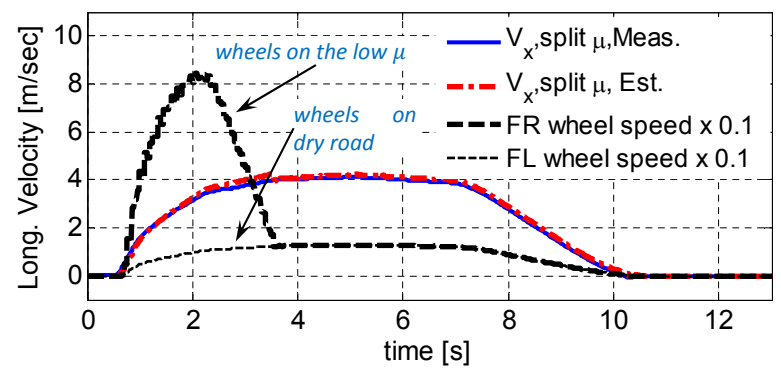

Fig. 9: Longitudinal velocity estimation for $A W D$, split $\mu$ on ice and dry

Figure 9 demonstrates good performance of the longitudinal velocity estimator validated by the measurement from the accurate GPS at the vehicle's CG. It also shows wheel speeds on dry and icy roads. Proper time-varying observer gains and track's weighted velocities lead to the observed smooth and accurate velocity estimation at the vehicle CG and consequently at corners for such high-slip conditions.

2) Steering on dry and snowy roads: Fig. 10 demonstrates velocity estimation results in a lane change (LC) on snow and ice for the $A W D$ configuration.

Fluctuations of the measured lateral acceleration in Fig. 10b and sudden changes of the vehicle yaw rate in Fig. 10c substantiate arduous characteristics of the driving scenario. Longitudinal and lateral velocity estimators' results show correspondence with the measured GPS data for such severe maneuver on a slippery surface and can be used for traction and stability control systems.

The provided experimental tests have been conducted so far on a vehicle with $A W D$ configuration. The proposed estimator can be utilized on vehicles with different driving axle configurations i.e. Rear and Forward-Wheel-Drive $(R W D, F W D)$. In order to evaluate the outcomes of the velocity estimator, a maneuver on a dry road with an oval shape has been performed and results are validated with the measured GPS velocities in Fig. 11

The measured accelerations and yaw rate in Fig. 11 resemble a harsh combined-slip maneuver with several oval steering and acceleration/deceleration, however the estimation outcome is accurate. 


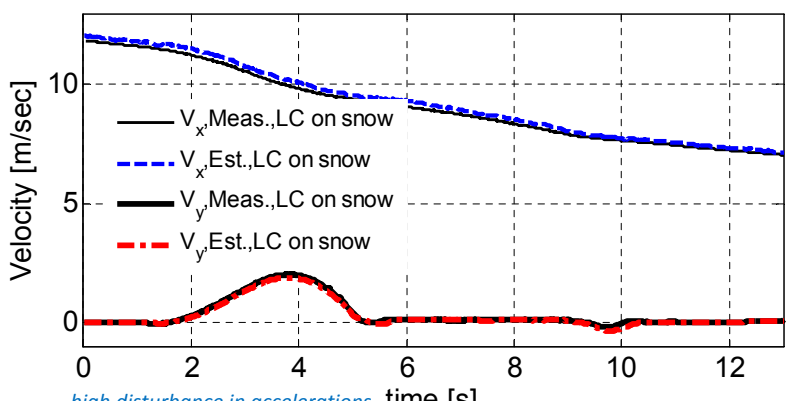

high disturbance in accelerations time [s]
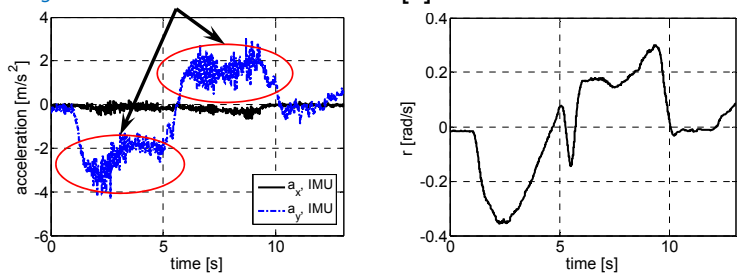

Fig. 10: Lane change (LC) on snow/ice, velocity estimation for $A W D$
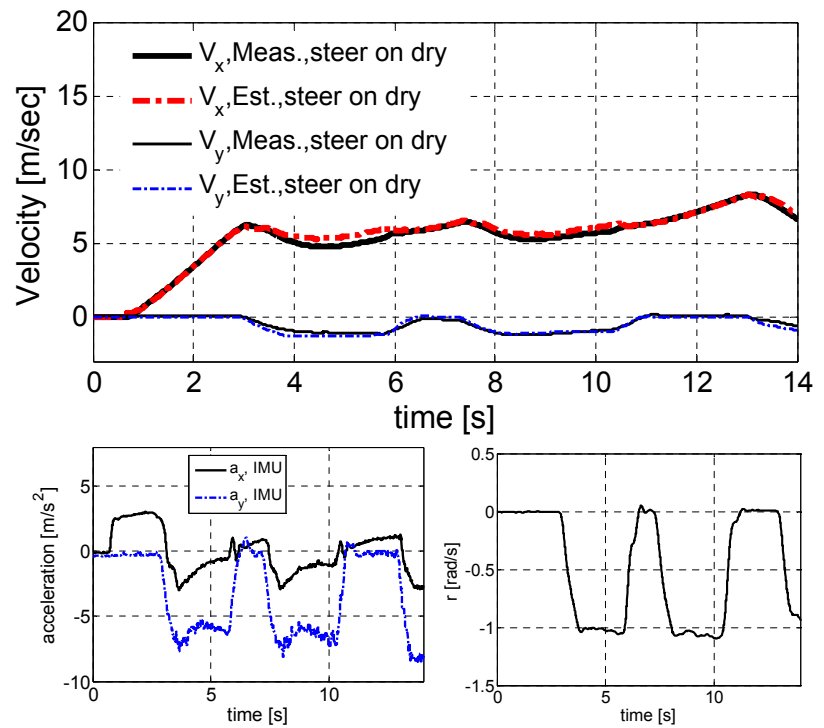

Fig. 11: Velocity estimation for RWD Config. on dry road

3) Acceleration-in-turn on dry and wet roads: To study the velocity estimator's performance in combined-slip conditions, an acceleration-in-turn (AiT) scenario with $A W D$ configuration is done on the dry road and results of the lateral velocity estimator are provided in Fig. 12

As can be seen from Fig. 12, high oscillations exist both in the lateral and longitudinal accelerations because of this harsh maneuver on a dry road and consequent high slip ratio regions, which reduce the lateral tire capacities significantly. The wheel speed and consequently the slip ratio increases significantly between $t=5$ and $t=7[s]$, but the proposed lateral state estimation methodology handles these situations and exhibit
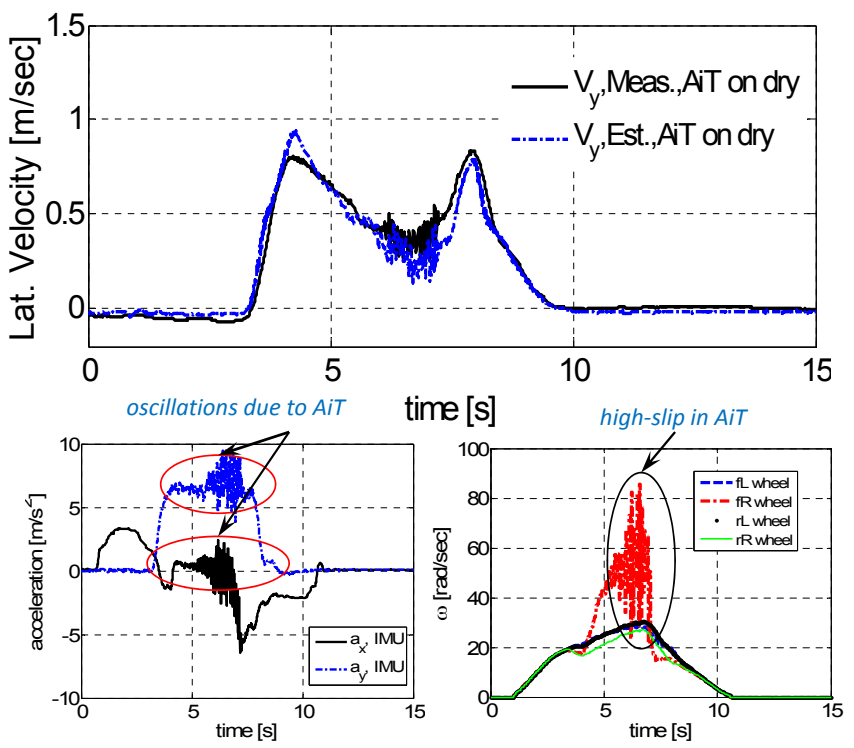

Fig. 12: Lateral velocity estimator for $A W D$, AiT on the dry road

smooth and accurate outcomes.

The next test is an acceleration during a large left steering from a wet and slippery surface with $\mu \approx 0.4$ to a dry surface with $R W D$ configuration. This test is done to explore the performance of the state estimators and incorporation of each track with the weights (34), (44) on a combined dry/wet surface. Validated estimation results with the GPS data, measured accelerations, and yaw rate are shown in Fig. 13.
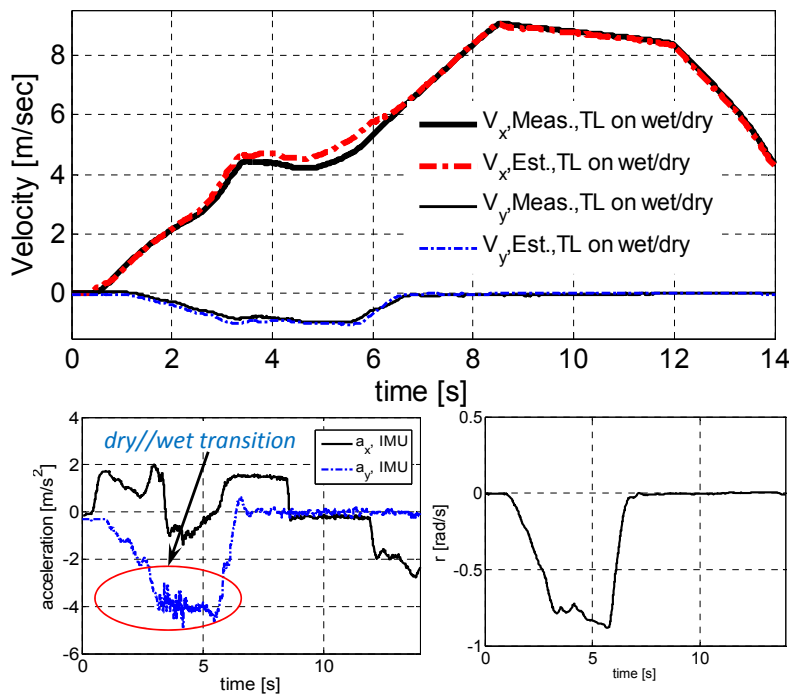

Fig. 13: Acceleration with large left turn (TL) on wet/dry, $R W D$

The maneuver is demanding because of the reduction effect of the longitudinal slip on the tire lateral capacity and the transition between the dry and slippery surfaces, but the 
estimators provide smooth results because of the weighted tracks' velocities and time-varying observer gains.

Performance of the longitudinal and lateral velocity estimators are investigated in Table III for vehicle with $A W D$ and $R W D$ traction configurations in different driving conditions.

TABLE III: NRMS of the Errors for the Velocity Estimators at Vehicle's CG for AWD and RWD Configurations

\begin{tabular}{l|cccc}
\hline \multirow{2}{*}{ Maneuvers } & \multicolumn{3}{|c}{ Estimated Vx } & \multicolumn{2}{c}{ Estimated Vy } \\
\cline { 2 - 5 } & $\varepsilon_{n}[\%]$ & $s_{m}[m / s]$ & $\varepsilon_{n}[\%]$ & $s_{m}[m / s]$ \\
\hline \hline $\boldsymbol{A W D}$ & \multicolumn{4}{c}{} \\
\hline Launch on ice/dry, split $\mu$ & 2.64 & 4.14 & 0.2 & 0.08 \\
AiT on dry & 4.61 & 9.1 & 6.3 & 0.83 \\
Steering \& Accel./Deccel., snow & 3.38 & 9.98 & 4.11 & 4.04 \\
LC on snow & 1.6 & 11.86 & 3.29 & 2.02 \\
\hline $\boldsymbol{R W D}$ & & & & \\
\hline Oval steering on dry & 3.15 & 8.38 & 7.6 & 1.24 \\
Accel. \& left turn dry/wet & 2.07 & 9.06 & 5.91 & 0.95 \\
Full turn on dry & 0.65 & 9.01 & 7.15 & 0.52 \\
Full turn on dry/wet & 0.79 & 7.51 & 6.21 & 0.74 \\
\hline
\end{tabular}

The corner-based state estimators with the adaptive weighted velocity mapping modules (34), (43) based on the slip ratio/angle can handle dry and slippery roads with error NRMS $\varepsilon_{n}<7.4 \%$ for the force and error NRMS $\varepsilon_{n}<6.3 \%$ for the velocity estimation. The state estimators exhibit reliable performance for the maneuver with the pure-slip characteristics (i.e. launch, normal driving, acceleration/deceleration) as well as the combined-slip ones (i.e. acceleration-in-turn and brakein-turn), in which the tire capacities reduces significantly both in the lateral and longitudinal directions.

\section{CONCLUSION}

This paper has proposed a method to estimate the vehicle states at each corner robust to road conditions. An observer is provided on the wheel dynamics for the longitudinal force estimation at each corner and its stability is discussed. Lateral tire forces are estimated utilizing UKF on the handling dynamics and without any assumption on the lateral force distribution. Vertical tire forces were calculated at each corner employing the roll and pitch effects of the vehicle sprung mass. To tackle the limitations of the kinematic and modelbased velocity estimation approaches, the average Lumped LuGre model and the kinematics are coupled at each corner to estimate the longitudinal and lateral velocities.

Several road experiments with normal and harsh driving conditions have been conducted on dry and slippery roads to validate the approach. Based on the road experiment results on an instrumented vehicle, the following conclusions can be made:

The presented corner-based longitudinal/lateral force and velocity estimation robust to the road conditions is an advantage over estimators on double track models because it can exhibit saturation and capacity conditions of all tires. The proposed force estimator does not implement any tire model and is independent from changes in the road friction or tire parameters due to wear, inflation pressure, temperature, etc. In addition, the suggested UKF-based lateral force estimators can address the cases in which tires are on surfaces with various road friction.

Utilizing the dynamics on the LuGre's internal deflection states, the proposed corner-based velocity estimators form a linear parameter-varying model with the road friction as uncertainties. The stability of the velocity estimators' error dynamics is investigated with the affine quadratic stability approach. An observer was designed as well with variable gains with respect to the wheel speed. One significant advantage of the suggested velocity estimator is that a unidirectional lumped LuGre model could be used instead of the combined one since the term containing the combined friction model i.e. $\frac{\sigma_{0 q}\left|V_{r q}\right|}{\theta g\left(V_{r q}\right)} \bar{z}_{q}$ was considered as uncertainty.

The results of the road experiments on different road conditions substantiate that the algorithm can handle different vehicle traction configurations i.e. $A W D$ and $R W D$. This preeminence of the algorithm makes it appropriate for a wide range of vehicles' traction configurations.

The algorithm can be integrated with various active safety systems (e.g. stability control, traction control, and roll over prevention), or road angle estimators, to ensure reliable performance of such systems in presence of model uncertainties and road friction changes. In addition, while preserving the overall structure of the estimation, one can replace or modify estimators independently because of the modularity of the developed structure.

Observed inconsistencies in some tests of the proposed estimation approach are due to the lack of a general chassis model, a limitation to be addressed in future work, using the vehicle generalized force model. Moreover, wheel torques need to be estimated using accelerations and engine/brake torques to be able to utilize the proposed estimation structure for conventional vehicles.

Note for the esteemed reviewer(s): The conclusions on performance of the observer in presence of faulty measurements had been removed from here on the previously revised manuscript.

\section{ACKNOWLEDGMENT}

The authors would like to acknowledge the financial support of the Automotive Partnership Canada, Ontario Research Fund, and the financial and technical support of General Motors Co.

\section{REFERENCES}

[1] N. Patel, C. Edwards, and S. Spurgeon, "Tyreroad friction estimationa comparative study," Proceedings of the Institution of Mechanical Engineers, Part D: Journal of Automobile Engineering, vol. 222, no. 12, pp. 2337-2351, 2008.

[2] G. Baffet, A. Charara, and D. Lechner, "Estimation of vehicle sideslip, tire force and wheel cornering stiffness," Control Engineering Practice, vol. 17, no. 11, pp. 1255-1264, 2009.

[3] R. Rajamani, G. Phanomchoeng, D. Piyabongkarn, and J. Y. Lew, “Algorithms for real-time estimation of individual wheel tire-road friction coefficients," Mechatronics, IEEE/ASME Transactions on, vol. 17, no. 6, pp. 1183-1195, 2012.

[4] M. Doumiati, A. Victorino, D. Lechner, G. Baffet, and A. Charara, "Observers for vehicle tyre/road forces estimation: experimental validation," Vehicle System Dynamics, vol. 48, no. 11, pp. 1345-1378, 2010. 
[5] W. Cho, J. Yoon, S. Yim, B. Koo, and K. Yi, "Estimation of tire forces for application to vehicle stability control," Vehicular Technology, IEEE Transactions on, vol. 59, no. 2, pp. 638-649, 2010.

[6] E. Hashemi, A. Kasaeizadeh, A. Khajepour, N. Mushchuk, and S.-K. Chen, "Robust Estimation and Experimental Evaluation of Longitudinal Friction Forces in Ground Vehicles," in ASME IMECE2014, 2014.

[7] S. Mammar, S. Glaser, and M. Netto, "Vehicle lateral dynamics estimation using unknown input proportional-integral observers," in American Control Conference, 2006. IEEE, 2006, pp. 6-pp.

[8] Y. Wang, D. M. Bevly, and S.-k. Chen, "Longitudinal Tire Force Estimation with Unknown Input Observer," in ASME DSCC2012, 2012.

[9] _ - "Lateral tire force estimation with unknown input observer," in ASME 2012 5th Annual Dynamic Systems and Control Conference joint with the JSME 2012 11th Motion and Vibration Conference. American Society of Mechanical Engineers, 2012, pp. 531-538.

[10] Y.-H. J. Hsu, "Estimation and control of lateral tire forces using steering torque," Ph.D. dissertation, Stanford University, 2009.

[11] M. Doumiati, A. C. Victorino, A. Charara, and D. Lechner, "Onboard real-time estimation of vehicle lateral tire-road forces and sideslip angle," IEEE/ASME Transactions on Mechatronics, vol. 16, no. 4, pp. 601-614, 2011.

[12] X. Gao and Z. Yu, "Vehicle sideslip angle estimation by using high gain observer," in AVEC 9th International Symposium on Advanced Vehicle Control, 2008, pp. 509-514.

[13] G. Baffet, A. Charara, and G. Dherbomez, "An observer of tire-road forces and friction for active security vehicle systems," Mechatronics, IEEE/ASME Transactions on, vol. 12, no. 6, pp. 651-661, 2007.

[14] J. Ryu, "State and Parameter Estimation for Vehicle Dynamics Control using GPS," Ph.D. dissertation, Stanford University, 2004.

[15] J. Ryu and J. C. Gerdes, "Integrating inertial sensors with global positioning system (gps) for vehicle dynamics control," Journal of Dynamic Systems, Measurement, and Control, vol. 126, no. 2, pp. 243254, 2004.

[16] L. Imsland, T. A. Johansen, T. I. Fossen, H. F. Grip, J. C. Kalkkuhl, and A. Suissa, "Vehicle velocity estimation using nonlinear observers," Automatica, vol. 42, no. 12, pp. 2091-2103, 2006.

[17] J. A. Farrell, H.-S. Tan, and Y. Yang, "Carrier phase gps-aided ins-based vehicle lateral control," Journal of Dynamic Systems, Measurement, and Control, vol. 125, no. 3, pp. 339-353, 2003.

[18] D. M. Bevly, "Global positioning system (gps): A low-cost velocity sensor for correcting inertial sensor errors on ground vehicles," Journal of dynamic systems, measurement, and control, vol. 126, no. 2, pp. 255-264, 2004.

[19] D. M. Bevly, J. Ryu, and J. C. Gerde, "Integrating ins sensors with gps measurements for continuous estimation of vehicle sideslip, roll, and tire cornering stiffness," Intelligent Transportation Systems, IEEE Transactions on, vol. 7, no. 4, pp. 483-493, 2006.

[20] J.-H. Yoon and H. Peng, "A cost-effective sideslip estimation method using velocity measurements from two gps receivers," Vehicular Technology, IEEE Transactions on, vol. 63, no. 6, pp. 2589-2599, 2014.

[21] Y. Fukada, "Slip-angle estimation for vehicle stability control," Vehicle System Dynamics, vol. 32, no. 4, pp. 375-388, 1999.

[22] A. Hac and M. D. Simpson, "Estimation of vehicle side slip angle and yaw rate," SAE Technical Paper, Tech. Rep., 2000.

[23] L. Imsland, H. F. Grip, T. A. Johansen, T. I. Fossen, J. C. Kalkkuhl, and A. Suissa, "Nonlinear observer for vehicle velocity with friction and road bank angle adaptation-validation and comparison with an extended kalman filter," SAE Technical Paper, Tech. Rep., 2007.

[24] M. Satria and M. Best, "Comparison between Kalman Filter and Robust Filter for Vehicle Handling Dynamics State Estimation," in $S A E$ Technical Paper 2002-01-1185, 2002.

[25] T. a. Wenzel, K. J. Burnham, M. V. Blundell, and R. a. Williams, "Dual Extended Kalman Filter for Vehicle State and Parameter Estimation," Vehicle System Dynamics, vol. 44, no. 2, pp. 153-171, 2006.
[26] L. Tong, "An Approach for Vehicle State Estimation Using Extended Kalman Filter," in Syst. Simul. Sci. Comput., vol. 1, 2012, pp. 56-63.

[27] M. Burckhardt, Fahrwerktechnik: Radschlupfregelsysteme, Germany, 1993.

[28] X. Huang and J. Wang, "Robust Sideslip Angle Estimation for Lightweight Vehicles Using Smooth Variable Structure Filter," in ASME Dynamic Systems and Control Conference, 2013, pp. 1-8.

[29] S. Julier and J. Uhlmann, "A New Extension of the Kalman Filter to Nonlinear Systems," in The 11th International Symposium on Aerospace/Defense Sensing, Simulation and Controls, 1997.

[30] S. Antonov, A. Fehn, and A. Kugi, "Unscented Kalman Filter for Vehicle State Estimation," Vehicle System Dynamics, vol. 49, no. 9, pp. 1497-1520, 2011.

[31] H. B. Pacejka and I. J. M. Besselink, "Magic Formula Tyre Model with Transient Properties," Vehicle System Dynamics, vol. 27, pp. 234-249, 1997.

[32] M. Wielitzka, M. Dagen, and T. Ortmaier, "State estimation of vehicle's lateral dynamics using unscented kalman filter," in Decision and Control (CDC), 2014 IEEE 53rd Annual Conference on. IEEE, 2014, pp. 50155020 .

[33] F. Sun, K. Lolenko, and J. Rudolph, "Nonlinear observer design for state estimation during antilock braking," Proceedings of the Institution of Mechanical Engineers, Part I: Journal of Systems and Control Engineering, vol. 228, no. 2, pp. 78-86, 2014.

[34] C. Canudas-De-Wit and P. Tsiotras, "Dynamic Tire Friction Models for Vehicle Traction Control," in proceedings of the 38th Conference on Decision \& Control, 1999, pp. 3746-3751.

[35] H. F. Grip, L. Imsland, T. A. Johansen, T. I. Fossen, J. C. Kalkkuhl, and A. Suissa, "Nonlinear vehicle side-slip estimation with friction adaptation,” Automatica, vol. 44, no. 3, pp. 611-622, 2008.

[36] H. Faer Grip, L. Imsland, T. A. Johansen, J. C. Kalkkuhl, and A. Suissa, "Vehicle sideslip estimation: Design, implementation, and experimental validation," IEEE control systems, vol. 29, no. 5, pp. 36-52, 2009.

[37] S.-H. You, J.-O. Hahn, and H. Lee, "New adaptive approaches to real-time estimation of vehicle sideslip angle," Control Engineering Practice, vol. 17, no. 12, pp. 1367-1379, 2009.

[38] G. Magallan, C. H. D. Angelo, and G. O. García, "Maximization of the traction forces in a 2WD electric vehicle," Vehicular Technology, IEEE Transactions on, vol. 60, no. 2, pp. 369-380, 2011.

[39] X. Zhang, Y. Xu, M. Pan, and F. Ren, "A vehicle ABS adaptive slidingmode control algorithm based on the vehicle velocity estimation and tyre/road friction coefficient estimations," Vehicle System Dynamics, vol. 52, no. 4, pp. 475-503, 2014.

[40] F. Sun, X. Huang, J. Rudolph, and K. Lolenko, "Vehicle state estimation for anti-lock control with nonlinear observer," Control Engineering Practice, vol. 43, pp. 69-84, 2015.

[41] Y.-H. J. Hsu, S. M. Laws, and J. C. Gerdes, "Estimation of tire slip angle and friction limits using steering torque," IEEE Transactions on Control Systems Technology, vol. 18, no. 4, pp. 896-907, 2010.

[42] K. Nam, H. Fujimoto, and Y. Hori, "Lateral stability control of in-wheelmotor-driven electric vehicles based on sideslip angle estimation using lateral tire force sensors," Vehicular Technology, IEEE Transactions on, vol. 61, no. 5, pp. 1972-1985, 2012.

[43] M. Gadola, D. Chindamo, M. Romano, and F. Padula, "Development and validation of a kalman filter-based model for vehicle slip angle estimation," Vehicle System Dynamics, vol. 52, no. 1, pp. 68-84, 2014.

[44] C. Ahn, H. Peng, and H. E. Tseng, "Robust estimation of road frictional coefficient," Control Systems Technology, IEEE Transactions on, vol. 21, no. 1, pp. 1-13, 2013.

[45] R. Wang and J. Wang, "Tire-road friction coefficient and tire cornering stiffness estimation based on longitudinal tire force difference generation," Control Engineering Practice, vol. 21, no. 1, pp. 65-75, 2013.

[46] E. Hashemi, M. Pirani, A. Khajepour, B. Fidan, A. Kasaiezadeh, S. Chen, and B. Litkouhi, "Integrated estimation structure for the tire 
friction forces in ground vehicles," in Advanced Intelligent Mechatronics, 2016 IEEE Conference on. IEEE, 2016.

[47] H. Berghuis and H. Nijmeijer, "A passivity approach to controllerobserver design for robots," Robotics and Automation, IEEE Transactions on, vol. 9, no. 6, pp. 740-754, 1993.

[48] Y. Choi, "Pid state observer for robotic systems," in American Control Conference, 2006. IEEE, 2006, pp. 6-pp.

[49] A.-G. Wu, G.-R. Duan, and Y.-M. Fu, "Generalized pid observer design for descriptor linear systems," Systems, Man, and Cybernetics, Part B: Cybernetics, IEEE Transactions on, vol. 37, no. 5, pp. 1390-1395, 2007.

[50] J. Y. Peng and X. B. Chen, "Integrated pid-based sliding mode state estimation and control for piezoelectric actuators," Mechatronics, IEEE/ASME Transactions on, vol. 19, no. 1, pp. 88-99, 2014.

[51] E. Hashemi, R. Zarringhalam, A. Khajepour, W. Melek, A. Kasaiezadeh, and S.-K. Chen, "Real-time estimation of the road bank and grade angles with unknown input observers," Vehicle system dynamics, pp. 1-20, 2017.

[52] L. M. Silverman and H. Meadows, "Controllability and observability in time-variable linear systems," SIAM Journal on Control, vol. 5, no. 1, pp. 64-73, 1967.

[53] R. Tóth, Modeling and identification of linear parameter-varying systems. Springer, 2010, vol. 403.

[54] R. V. D. Merwe and E. Wan, "Efficient Derivative-Free Kalman Filters for Online Learning." in European Symposium on Artificial Neural Networks, ESANN, 2001, pp. 205-210.

[55] G. F. Franklin, J. D. Powell, and M. L. Workman, Digital Control of Dynamic Systems. Menlo Park: Addison Wesley Longman, 1998.

[56] E. Haykin, Simon, Kalman Filtering and Neural Networks. John Wiley \& Sons, Inc., 2004, vol. 47.

[57] F. Gustafsson and G. Hendeby, "Some Relations Between Extended and Unscented Kalman Filters," Signal Processing, IEEE Transactions on, vol. 60, no. 2, pp. 545-555, 2012.

[58] C. Canudas-de Wit, P. Tsiotras, E. Velenis, M. B. Gissinger, and G. Gissinger, "Dynamic Friction Models for Road/Tire Longitudinal Interaction," Vehicle System Dynamics, vol. 39, pp. 189-226, 2003.

[59] E. Hashemi, M. Pirani, A. Khajepour, and A. Kasaiezadeh, "A comprehensive study on the stability analysis of vehicle dynamics with pure/combined-slip tyre models," Vehicle system dynamics, vol. 54, no. 12, pp. 1736-1761, 2016.

[60] Y. Chen and J. Wang, "Adaptive vehicle speed control with input injections for longitudinal motion independent road frictional condition estimation," Vehicular Technology, IEEE Transactions on, vol. 60, no. 3 , pp. 839-848, 2011.

[61] Y. Wang and D. Bevly, "Longitudinal Vehicle State Estimation; General Motors Summary Report," General Motors Summary Report, Tech. Rep., 2010.

[62] P. Gahinet, P. Apkarian, and M. Chilali, "Affine Parameter-Dependent Lyapunov Functions and Real Parametric Uncertainty," IEEE Transactions on Automatic Control, vol. 41, no. 3, pp. 436-442, 1996.

[63] J. Lofberg, "Yalmip: A toolbox for modeling and optimization in matlab," in Computer Aided Control Systems Design, 2004 IEEE International Symposium on. IEEE, 2004, pp. 284-289. 